\title{
GENERATORS AND BERNOULLIAN FACTORS FOR AMENABLE ACTIONS AND COCYCLES ON THEIR ORBITS
}

\author{
Alexandre I. Danilenko and Kyewon K. Park
}

\begin{abstract}
Using the orbital approach to the entropy theory we extend from $\mathbb{Z}$ actions to general countable amenable group actions $T$ (or provide new short proofs to) the following results: (1) relative and absolute Krieger theorem about finite generating partitions (and its infinite Rokhlin counterpart in case $h(T)=\infty$ ), (2) relative and absolute Sinai theorem about Bernoullian factors, (3) Thouvenot theorem that every intermediate factor of a relatively Bernoullian action is also relatively Bernoullian, (4) Thouvenot theorem that a factor of $T$ with the strong Pinsker property enjoys this property, (5) Smorodinsky-Thouvenot theorem that $T$ can be spanned by three Bernoullian factors, (6) Ornstein-Weiss isomorphism theory for Bernoullian actions of the same entropy (provided that they possess generating partitions with at least 3 elements), (7) there are uncountably many non-equivalent CPE extensions of $T$ of the same relative entropy, etc.

In proving these theorems, we were able to bypass the machinery from [OrW] except of the Rokhlin lemma. It is shown that the language of orbit equivalence relations and their cocycles (unlike the standard dynamical one) is well suited for the inducing operation needed to settle (1), (5) and (6).
\end{abstract}

\section{INTRODUCTION}

The purpose of the present paper is to extend the classical entropy theory to the framework of general amenable actions by exploiting the language of equivalence relations and their cocycles. This method enables us to bypass the machinary of $[\mathrm{OrW}]$. Thus our work is a natural continuation of the previous paper [Da3] of the first named author. While most of the principal results in [Da3] are about the Pinsker algebras and CPE actions, our research here is concerned mainly with Bernoullian factors and generating partitions for actions of countable amenable groups (both in the relative and absolute setting) and related topics.

Recall that the entropy theory of general amenable group actions was initiated in [Ki] and developed later in depth for isomorphism theorems in [OrW] (see also [Ol]). Recently Rudolph and Weiss [RW] applied the orbit theory to settle a (difficult) problem of purely entropic nature: amenable actions of completely positive entropy (CPE) are uniformly mixing. The way the orbit theory was involved in [RW] (as well as in [Da3] and the present paper) comes from a crucial observation that the relative entropy of an amenable process is invariant under the factor orbit equivalence. Recall that two triplets of the form an amenable group, an ergodic action of this group and a factor of this action $\}$ are factor orbit equivalent if there exists an orbit equivalence which "respects" the factors. Since the actions of countable

1991 Mathematics Subject Classification. Primary 37A35, 37A20; Secondary 37A15.

Key words and phrases. Discrete equivalence relation, cocycle, amenable group action, entropy, Bernoullian factor.

The first named author was supported in part by CRDF grant UM1-2092 and the second named author was supported in part by BK21 grant. 
amenable groups have the same orbit structure like a $\mathbb{Z}$-action $[\mathrm{CFW}]$, we thus can establish some "relative entropy" theorems for general amenable actions by deducing them directly from their classical (i.e. $\mathbb{Z}$-) counterparts. Recall that the standard approach requires an independent proof by following the classical one.

This orbital approach in the entropy theory was further investigated in [Da3] which leads, among others, to new short (orbital) proofs of the main theorems from [WaZ] and [GTW]. Moreover, the cited above results of Rudolph and Weiss were reproved there in more algebraic language of measurable equivalence relations and their cocycles, independent of the Rokhlin lemma, Shannon-McMillan theorem, ergodic theorems for amenable actions, etc. i.e. the standard machinery from [OrW] or $[\mathrm{Ki}]$.

Now we state the main results of this work together with some comments and short historical remarks (we excuse for their incompleteness). The proofs will be given in Sections $2-7$. The precise definitions and preliminary material from the orbit theory and the entropy theory for amenable actions will be given in Section 1.

The Kolmogorov-Sinai theorem says that the entropy of a measure preserving transformation is equal to its entropy with respect to any generating partition. In [Rok], Rokhlin proved the existence of countable generating partition for every aperiodic transformation. This result was later strengthened by Krieger [Kr]: if the entropy of an ergodic transformation is less than $\log k$ then there is a finite generator of cardinality $k$. The Krieger theorem was extended to $\mathbb{Z}^{d}$-actions with positive entropy by Thouvenot [Th1, Proposition 6] but the cardinality of his generator is $k+1$ (see also $[\mathrm{KaW}]$ ). Finally Rosental extended the Krieger theorem to the ergodic free actions of general countable amenable groups. For every such action, he constructed in [Ro] some special-uniform-generator of cardinality $k+2$.

We first demonstrate the relative versions of the Kolmogorov-Sinai, Krieger and Rokhlin theorems for general amenable actions as follows:

Theorem 2.7. Let $T$ be an ergodic action of a countable amenable group and $\mathfrak{F}$ a class-bijective factor of $T$.

(i) If $P$ is a finite $\mathfrak{F}$-relative generator for $T$ then $h(T, P \mid \mathfrak{F})=h(T \mid \mathfrak{F})$.

(ii) If $h(T \mid \mathfrak{F})<\log k$ then there is a finite $\mathfrak{F}$-relative generator $P$ for $T$ with $\# P=k$.

(iii) If $h(T \mid \mathfrak{F})=\infty$ then there is a countable $\mathfrak{F}$-relative generator for $T$.

We say that a factor is class-bijective if the corresponding factor map does not collapse the points on the same $T$-orbit. We remark that Kifer and Weiss proved recently (i) and (ii) for $\mathbb{Z}$-actions in $[\mathrm{KW}]$. Their proof is quite different in the sense that it is longer and uses some classical technique that is specific to $\mathbb{Z}$-actions. On the other hand their proof is independent of $[\mathrm{Kr}]$ and $[\mathrm{RW}]$.

From Theorem 2.7 and some other facts (to be explained below) we deduce (at the very end of the paper) its absolute counterpart:

Theorem 7.2. Let $T$ be an ergodic free $G$-action. If $0<h(T)<\log k$ for some integer $k$ then there exists a finite generator $P$ of $T$ with $\# P=k$ if $k$ is not prime and $k+1$ otherwise. If $h(T)=\infty$ then there exists a countable generating partition for $T$.

Thus we improve the upper estimation for the cardinality of the generators obtained in [Ro]. 
The Ornstein isomorphism theory was relativised by Thouvenot in [Th1] (see also [Li] for the infinite entropy case). It was extended later from $\mathbb{Z}$-actions to $\mathbb{R}$ and $\left(\sum_{n=1}^{\infty} \mathbb{Z} / 2 \mathbb{Z}\right)$-actions in $[\mathrm{Fi}]$ and $[\mathrm{FiH}]$ respectively. Ornstein and Weiss ask in $[\mathrm{OrW}]$ if it is possible to extend the Thouvenot relative theory to actions of general amenable groups? We give a partial answer to this question affirmatively by demonstrating

Theorem 4.9. Let $T$ be an ergodic action of $G$ and $\mathfrak{F} \subset \mathfrak{H}$ two factors of $T$. If $\mathfrak{F}$ is class-bijective and $T$ is $\mathfrak{F}$-relatively Bernoullian then so is $T \uparrow \mathfrak{H}$.

and

Corollary 4.16. Every two relatively Bernoullian extensions of $T$ with the same relative entropy are equivalent.

We say that $T$ (or a $T$-factor containing $\mathfrak{F}$ ) is $\mathfrak{F}$-relatively Bernoullian if it splits into Cartesian product of $\mathfrak{F}$ and some complementary Bernoullian factor of $T$. We show in Proposition 3.8 that the property of relative Bernoulicity is invariant under the factor orbit equivalence. This is true for general - not necessarily amenablecountable group actions.

Of course the above two claims can be restated in the equivalent language of relative finitely determined (FD) processes and we conclude, in particular, that the relative FD-property is invariant under the factor orbit equivalence. However we would not explore the relative FD concept since we achieve our results like Theorem 4.9 and Corollary 4.16 independent of it. It is worthwhile to remark that we establish Corollary 4.16 without applying the Ornstein-Weiss isomorphism theorem (or any other technique from $[\mathrm{OrW}]$ ). Just the contrary, we will deduce the isomorphism theorem from it (see Theorem 7.1 below).

We also extend another result of Thouvenot [Th2] to amenable actions. Recall that a $G$-action $T$ satisfies the strong Pinsker property if it is isomorphic to the Cartesian product of a Bernoullian factor and a 0-entropy factor $\mathfrak{E}$.

Theorem 4.12. Let $T$ be an ergodic free $G$-action satisfying the strong Pinsker property and $\mathfrak{F}$ a factor of $T$ such that the factor $\mathfrak{F} \cap \mathfrak{E}$ is class bijective. Then $T \nmid \mathfrak{F}$ satisfies the strong Pinsker property.

Next, we extend the Sinai theorem about Bernoullian factors to general amenable actions both in the relative and absolute setting.

Corollary 4.6 (see also Proposition 4.5). Given a class-bijective factor $\mathfrak{F}$ of a free ergodic action $T$ of $G$ and a finite distribution $I$ such that $H(I) \leq h(T \mid \mathfrak{F})$, there exists a Bernoullian factor $\mathfrak{E} \perp \mathfrak{F}$ having a finite Bernoullian generator $Q$ with dist $Q=I$. The same is true for a countable distribution $I$, and if $H(I)=\infty$ we can claim in addition that $h\left(T \mid \mathfrak{F} \vee \bigvee_{g \in G} T_{g} Q\right)=0$.

Theorem 5.5. Let $T$ be an ergodic G-action. Then for each finite or countable distribution $I$ with $H(I) \in(0, h(T))$ there exists a Bernoullian factor $\mathfrak{F}$ of $T$ with a Bernoullian generator $Q$ such that $\operatorname{dist} Q=I$. Moreover, there is a Bernoullian $T$-factor $\mathfrak{H}$ of the full entropy, i.e. $h(T \mid \mathfrak{H})=0$. (Notice that in case $h(T)<\infty$ the last formula is equivalent to $h(T, \mathfrak{H})=h(T)$.)

Note that our proof of Theorem 5.5 is much shorter that the original one from [OrW]. 
Ornstein and Shields constructed in $[\mathrm{OrS}]$ an example of uncountably many non-conjugate CPE-transformations of the same entropy. Then Swanson [Sw] and Hoffman $[\mathrm{Ho}]$ produced a relative version of this example. We extend this relative version to general amenable actions as follows.

Proposition 4.15(ii). Let $T$ be an ergodic free action of $G$. Then there exist uncountably many non-equivalent relatively CPE extensions of $T$ with the same entropy.

Following $[\mathrm{Ho}]$ we also show

Proposition 4.18 (ii). Given an ergodic free action $T$ of $G$, there exists an ergodic extension of $T$ which is relatively prime.

Notice that even in the case $G=\mathbb{Z}$ our results (Propositions 4.15(ii) and 4.18(ii)) are different from those of Hoffman. They are more general in what our $T$ is arbitrary ergodic instead of only Bernoullian in [Ho]. But on the other hand they are a bit weaker, since we do not control the "absolute properties" of the extensions of $T$ while in [Ho] the extensions are proved to be Bernoullian.

In [STh] Smorodinsky and Thouvenot proved that every ergodic transformation of positive entropy can be spanned by three Bernoullian factors. This was extented to $\mathbb{R}$-actions in $[\mathrm{Pa}]$. Using the orbit technique we are now able to prove

Theorem 6.1. Let $T$ be an ergodic action of a countable amenable group $G$ on $\left(X, \mathfrak{B}_{X}, \mu\right)$ with $0<h(T) \leq \infty$. Then for every $\epsilon>0$, there are three Bernoullian factors $\mathfrak{F}_{0}, \mathfrak{F}_{1}$ and $\mathfrak{F}_{2}$ of $T$ such that $\mathfrak{F}_{0} \vee \mathfrak{F}_{1} \vee \mathfrak{F}_{2}=\mathfrak{B}_{X}$ and $h\left(T \uparrow \mathfrak{F}_{i}\right)<\epsilon, i=1,2$.

The crucial point of the original proof in [STh] is to consider some special transformation induced by $T$. However the inducing on a subset is not possible for actions of groups other than $\mathbb{Z}$. Nevertheless, it is well defined for equivalence relations and their cocycles. We study the properties of such an inducing in Lemma 6.4. Then we show how it works to complete the proof of Theorem 6.1. Remark that even in the case of $\mathbb{Z}$-actions our proof is simpler than that from [STh], since we do not use directly the successive approximation argument which is the most technical ingredient of the Smorodinsky-Thouvenot paper.

Furthermore, the cocycle inducing technique is applied to prove in a short way Theorem 7.2 about absolute generators (we stated it above) and the following Ornstein-Weiss isomorphism theorem for countable amenable actions:

Theorem 7.1. Let $T$ and $T^{\prime}$ be two Bernoullian G-actions. They are conjugate if one of the following is satisfied:

(i) $h(T)=h\left(T^{\prime}\right)=\infty$,

(ii) $h(T)=h\left(T^{\prime}\right)<\infty$ and each of $T$ and $T^{\prime}$ has an independent generator with at least 3 elements (as a partition).

To prove Theorems 5.5, 6.1 and 7.2 we need an auxiliary fact that every ergodic free $G$-action of positive entropy has a free factor of arbitrarily small entropy. We present elementary proofs of this fact for several important classes of amenable groups, for instance: (a) $G$ is torsion free, (b) $G$ is Abelian, (c) $G$ is locally finite, etc. (see Proposition 5.1). However, to settle the general case (see Theorem 5.4) we use the Rokhlin lemma from [OrW]. 


\section{Notation. Preliminaries}

Orbital background. Let $\left(X, \mathfrak{B}_{X}, \mu\right)$ be a standard probability space. Throughout this paper we do not distinguish the objects (like subsets, maps, partitions, etc.) which agree on a $\mu$-conull subset. The trivial sub- $\sigma$-algebra of $\mathfrak{B}_{X}$ is denoted by $\mathfrak{N}_{X}$.

Let $\operatorname{Aut}(X, \mu)$ stand for the group of $\mu$-preserving invertible transformations of $X$. A measure preserving discrete equivalence relation $\mathcal{R}$ on $X$ is the orbit equivalence relation of a countable subgroup $G \subset \operatorname{Aut}(X, \mu)$ (see [FM] for details). This generating subgroup is highly non-unique. If $\# \mathcal{R}(x)<\infty$ a.e. we say that $\mathcal{R}$ is of type $I$.

$\mathcal{R}$ is hyperfinite if there exists a sequence $\mathcal{R}_{1} \subset \mathcal{R}_{2} \subset$. . of type $I$ subrelations of $\mathcal{R}$ with $\bigcup_{n} \mathcal{R}_{n}=\mathcal{R}$. The sequence $\left(\mathcal{R}_{n}\right)_{n}$ is called a filtration of $\mathcal{R}$. It follows from [Dy] that a measure preserving discrete equivalence relation is hyperfinite if and only if it is generated by a single transformation. The orbit equivalence relation of a measure preserving action of a countable amenable group is hyperfinite [CFW]. $\mathcal{R}$ is ergodic if every $\mathcal{R}$-invariant subset belongs to $\mathfrak{N}_{X}$. Any two ergodic hyperfinite measure preserving discrete equivalence relations are isomorphic in the natural sense (i.e. there exists an isomorphism of the underlying measure spaces that intertwines bijectively the corresponding equivalence classes) [Dy].

Everywhere below $\mathcal{R}$ is an ergodic measure preserving discrete equivalence relation on $\left(X, \mathfrak{B}_{X}, \mu\right)$. We let

$$
\begin{gathered}
{[\mathcal{R}]:=\{\gamma \in \operatorname{Aut}(X, \mu) \mid(x, \gamma x) \in \mathcal{R} \text { a.e. }\},} \\
N[\mathcal{R}]:=\{\theta \in \operatorname{Aut}(X, \mu) \mid \theta \mathcal{R}(x)=\mathcal{R}(\theta x) \text { a.e. }\}
\end{gathered}
$$

for the full group of $\mathcal{R}$ and the normalizer of $[\mathcal{R}]$ respectively. Clearly, $[\mathcal{R}]$ is a normal subgroup in $N[\mathcal{R}]$.

Let $A$ be a Polish group. A Borel map $\alpha: \mathcal{R} \rightarrow A$ is called a cocycle if

$$
\alpha\left(x, x^{\prime \prime}\right)=\alpha\left(x, x^{\prime}\right) \alpha\left(x^{\prime}, x^{\prime \prime}\right) \quad \text { for all }\left(x, x^{\prime}\right),\left(x^{\prime}, x^{\prime \prime}\right) \in \mathcal{R} .
$$

Two cocycles $\alpha, \beta: \mathcal{R} \rightarrow A$ are cohomologous if there is a Borel map $\phi: X \rightarrow A$ with

$$
\alpha\left(x, x^{\prime}\right)=\phi(x) \beta\left(x, x^{\prime}\right) \phi\left(x^{\prime}\right)^{-1} \quad \text { for all }\left(x, x^{\prime}\right) \in \mathcal{R} \cap(B \times B),
$$

where $B$ is a $\mu$-conull subset.

For a transformation $\theta \in N[\mathcal{R}]$, we define a cocycle $\alpha \circ \theta$ of $\mathcal{R}$ by setting

$$
\alpha \circ \theta\left(x, x^{\prime}\right)=\alpha\left(\theta x, \theta x^{\prime}\right)
$$

Two cocycles $\alpha, \beta: \mathcal{R} \rightarrow A$ are weakly equivalent if $\alpha$ is cohomologous to $\beta \circ \theta$ for a transformation $\theta \in N[\mathcal{R}]$. Clearly, the cohomology and the weak equivalence are equivalence relations on the set of $A$-valued cocycles of $\mathcal{R}$.

Let $\left(Y, \mathfrak{B}_{Y}, \nu\right)$ be another standard probability space. Given a cocycle $\alpha: \mathcal{R} \rightarrow$ Aut $(Y, \nu)$, we associate a measure preserving discrete equivalence relation $\mathcal{R}(\alpha)$ on $(X \times Y, \mu \times \nu)$ by setting

$$
(x, y) \sim_{\mathcal{R}(\alpha)}\left(x^{\prime}, y^{\prime}\right) \text { if }\left(x, x^{\prime}\right) \in \mathcal{R} \text { and } y^{\prime}=\alpha\left(x^{\prime}, x\right) y
$$


Then a one-to-one group homomorphism $[R] \ni \gamma \mapsto \gamma_{\alpha} \in[\mathcal{R}(\alpha)]$ is well defined via the formula

$$
\gamma_{\alpha}(x, y)=(\gamma x, \alpha(\gamma x, x) y),(x, y) \in X \times Y .
$$

The transformation $\gamma_{\alpha}$ is called the $\alpha$-skew product extension of $\gamma$. The equivalence relation $\mathcal{R}(\alpha)$ is called the $\alpha$-skew product extension of $\mathcal{R}$.

For a finite partition $P$ of $X \times Y$, we denote by $\left(P_{x}\right)_{x \in X}$ the associated measurable field of finite $Y$-partitions $\left(P_{x}\right.$ stands for the restriction of $P$ onto the $Y$-fiber over $x$ ). More generally, given a sub- $\sigma$-algebra $\mathfrak{F}$ of $\mathfrak{B}_{X} \otimes \mathfrak{B}_{Y}$, we denote by $\left(\mathfrak{F}_{x}\right)_{x \in X}$ the associated measurable field of sub- $\sigma$-algebras of $\mathfrak{B}_{Y}$. It is well known that the family of all sub- $\sigma$-algebras of $\mathfrak{B}_{Y}$ endowed with the strong operator topology is a Polish space (we identify a sub- $\sigma$-algebra with the orthogonal projector onto the corresponding $L^{2}(Y, \nu)$-subspace of invariant functions). Moreover, the map $X \ni x \mapsto \mathfrak{F}_{x}$ is measurable. Notice that for two sub- $\sigma$-algebras $\mathfrak{F}$ and $\mathfrak{F}^{\prime}$, we have $\mathfrak{F}_{x}=\mathfrak{F}_{x}^{\prime}$ for a.e. $x \in X$ if and only if $\mathfrak{F} \vee\left(\mathfrak{B}_{X} \otimes \mathfrak{N}_{Y}\right)=\mathfrak{F}^{\prime} \vee\left(\mathfrak{B}_{X} \otimes \mathfrak{N}_{Y}\right)$.

Definition 1.1. For a finite partition $P$ of $X \times Y$ and a type $I$ subrelation $\mathcal{S}$ of $\mathcal{R}$, we set

$$
h(\mathcal{S}, \alpha, P):=\int_{X} \frac{1}{\# \mathcal{S}(x)} H\left(\bigvee_{x^{\prime} \in \mathcal{S}(x)} \alpha\left(x, x^{\prime}\right) P_{x^{\prime}}\right) d \mu(x),
$$

and define the entropy of $(\alpha, P)$ as

$$
h(\alpha, P):=\inf \{h(S, \alpha, P) \mid \mathcal{S} \text { is a type I subrelation of } \mathcal{R}\} .
$$

For a sub- $\sigma$-algebra $\mathfrak{P}$ of $\mathfrak{B}_{X} \otimes \mathfrak{B}_{Y}$, we set

$$
h(\alpha, \mathfrak{P}):=\sup \{h(\alpha, P) \mid P \text { is a finite } \mathfrak{P} \text {-measurable partition of } X \times Y\} .
$$

The entropy of $\alpha$ is $h(\alpha):=h\left(\alpha, \mathfrak{B}_{X} \otimes \mathfrak{B}_{Y}\right)$. We say that $\alpha$ is $C P E$ (i.e. of completely positive entropy) if $h(\alpha, P)>0$ for every finite partition $P$ that is not contained in $\mathfrak{B}_{X} \otimes \mathfrak{N}_{Y}$.

It was shown in [Da3] that if $\mathcal{R}$ is hyperfinite and $\left(\mathcal{R}_{n}\right)_{n \geq 1}$ a filtration of $\mathcal{R}$ then the sequence $h\left(\mathcal{R}_{n}, \alpha, P\right)$ converges to $h(\alpha, P)$ as $n \rightarrow \infty$. This is true even without the ergodicity assumption on $\mathcal{R}$. In our case $-\mathcal{R}$ is ergodic - we can prove more.

Proposition 1.2. If $\mathcal{R}$ is hyperfinite then

$$
\frac{1}{\# \mathcal{R}_{n}(x)} H\left(\bigvee_{x^{\prime} \in \mathcal{R}_{n}(x)} \alpha\left(x, x^{\prime}\right) P_{x^{\prime}}\right) \rightarrow h(\alpha, P) \text { for a.a. } x
$$

as $n \rightarrow \infty$ for every filtration $\left(\mathcal{R}_{n}\right)_{n \geq 1}$ of $\mathcal{R}$.

Proof. Let $f_{n}(x):=\frac{1}{\# \mathcal{R}_{n}(x)} H\left(\bigvee_{x^{\prime} \in \mathcal{R}_{n}(x)} \alpha\left(x, x^{\prime}\right) P_{x^{\prime}}\right), x \in X, n>0$. It was shown in the proof of [Da3, Proposition 2.6] that $f_{n+1} \leq E\left(f_{n} \mid \mathcal{R}_{n+1}\right)$ a.e., where $E\left(. \mid \mathcal{R}_{n+1}\right)$ stands for the conditional expectation with respect to the $\sigma$-albebra of $\mathcal{R}_{n+1}$-invariant subsets. By the submartingales convergence theorem [Lo, §32], $f_{n}$ converges a.e. (and in $L^{1}(X, \mu)$ ) as $n \rightarrow \infty$. Since $f_{n}$ is $\mathcal{R}_{n}$-invariant, the limit function is $\mathcal{R}$-invariant and hence constant, say $c$. Then

$$
\int f_{n} d \mu=h\left(\mathcal{R}_{n}, \alpha, P\right) \rightarrow \int c d \mu=c
$$

and hence $c=h(\alpha, P)$. 
Entropy concepts for group actions. Let $G$ be a countable group, $T=\left\{T_{g}\right\}_{g \in G}$ an ergodic measure preserving action of $G$ on $\left(Y, \mathfrak{B}_{Y}, \nu\right)$. A $T$-invariant sub- $\sigma$ algebra $\mathfrak{E}$ of $\mathfrak{B}_{Y}$ is called a factor of $T$. The restriction of $T$ to $(\mathfrak{E}, \nu \uparrow \mathfrak{E})$ will be denoted by $T \nmid \mathfrak{E}$. We also call $T$ an extension of $T \uparrow \mathfrak{E}$.

A $T$-factor $\mathfrak{E}$ is class-bijective if for a measurable map $f: Y \rightarrow \mathbb{R}$ with $\mathfrak{E}=$ $f^{-1}\left(\mathfrak{B}_{\mathbb{R}}\right)$, we have that $f$ is one-to-one on the $T$-orbits a.e.. Clearly if $T$ is free then $\mathfrak{E}$ is class-bijective if and only if the factor-action $T \uparrow \mathfrak{E}$ is free (with respect to the measure $\nu \uparrow \mathfrak{E})$.

Given a cocycle $\beta$ of the $T$-orbit equivalence relation with values in $\operatorname{Aut}(Z, \kappa)$, denote by $T^{\beta}=\left\{\left(T_{g}\right)_{\beta}\right\}_{g \in G}$ the $\beta$-skew product extension of $T$. Then $\mathfrak{B}_{Y} \otimes \mathfrak{N}_{Z}$ is a class-bijective factor of $T^{\beta}$. Conversely, if $\mathfrak{E}$ is a class-bijective factor of an ergodic action $T$ then $T$ is isomorphic to a skew product extension of $T \uparrow \mathfrak{E}$.

Let $Q$ be a finite partition of $Y$ and $\mathfrak{E}$ a factor of $T$. The $\mathfrak{E}$-relative entropy of the process $(T, Q)$ is

$$
h(T, Q \mid \mathfrak{E}):=\inf \left\{\frac{1}{\# F} H\left(\bigvee_{g \in F} T_{g}^{-1} Q \mid \mathfrak{E}\right) \mid F \text { is a finite subset of } G\right\} .
$$

If $G$ is amenable then $h(T, Q \mid \mathfrak{E})=\lim _{i \rightarrow \infty} \frac{1}{\# F_{i}} H\left(\bigvee_{g \in F_{i}} T_{g}^{-1} Q \mid \mathfrak{E}\right)$ for each F $\varnothing$ lner sequence $\left(F_{i}\right)_{i \geq 1}$ in $G$ (see [Ol], [OrW], [Da3]).

For a sub- $\sigma$-algebra $\mathfrak{P}$ of $\mathfrak{B}_{Y}$ (not necessarily a factor of $T$ ), we set

$$
h(T, \mathfrak{P} \mid \mathfrak{E})=\sup \{h(T, Q \mid \mathfrak{E}) \mid Q \text { is a finite } \mathfrak{P} \text {-measurable partition of } Y\} .
$$

The $\mathfrak{E}$-relative entropy of $T$ is $h(T \mid \mathfrak{E}):=h\left(T, \mathfrak{B}_{Y} \mid \mathfrak{E}\right)$ and the $\mathfrak{E}$-relative Pinsker algebra of $T$ is

$$
\Pi(T \mid \mathfrak{E})=\vee\left\{Q \subset \mathfrak{B}_{Y} \mid h(T, Q \mid \mathfrak{E})=0\right\} .
$$

If $\Pi(T \mid \mathfrak{E})=\mathfrak{E}$ then $T$ is called $\mathfrak{E}$-relatively $C P E$. We will write $h(T), \Pi(T)$ and $h(T, \mathfrak{P})$ instead of $h\left(T \mid \mathfrak{N}_{Y}\right), \Pi\left(T \mid \mathfrak{N}_{Y}\right)$ and $h\left(T, \mathfrak{P} \mid \mathfrak{N}_{Y}\right)$ respectively.

It is easy to verify that $T$ is $\Pi(T)$-relatively CPE.

The next important statement is of constant use in the paper. Originally it was proved in $[\mathrm{RW}]$ in a different but equivalent form. Another purely orbital (independent of the machinery from [OrW]) proof of it is given in [Da3].

Lemma 1.3. Let $\alpha$ be a cocycle of a hyperfinite ergodic equivalence relation $\mathcal{R}$ on $\left(X, \mathfrak{B}_{X}, \mu\right)$ with values in Aut $(Y, \nu)$. If $T$ is a free $G$-action on $X$ generating $\mathcal{R}$ then

$$
h\left(T^{\alpha}, P \mid \mathfrak{B}_{X} \otimes \mathfrak{N}_{Y}\right)=h(\alpha, P)
$$

for every finite partition $P$ of $X \times Y$. This implies $h\left(T^{\alpha}, \mathfrak{P} \mid \mathfrak{B}_{X} \otimes \mathfrak{N}_{Y}\right)=h(\alpha, \mathfrak{P})$ for every sub- $\sigma$-algebra $\mathfrak{P} \subset \mathfrak{B}_{X} \otimes \mathfrak{B}_{Y}$. Moreover, $\alpha$ is $C P E$ if and only if $T^{\alpha}$ is $\left(\mathfrak{B}_{X} \otimes \mathfrak{N}_{Y}\right)$-relatively CPE.

\section{RELATIVE GENERATORS}

In this section we prove the relative Kolmogorov-Sinai, Krieger and Rokhlin theorems about generating partitions for amenable group actions (see Theorem 2.7). But first we establish their orbital counterparts.

Let $\left(X, \mathfrak{B}_{X}, \mu\right)$ and $\left(Y, \mathfrak{B}_{Y}, \nu\right)$ be standard probability spaces. Recall that throughout the paper $\mathcal{R}$ is an ergodic $\mu$-preserving countable equivalence relation on $X$ and $\alpha: \mathcal{R} \rightarrow \operatorname{Aut}(Y, \nu)$ a cocycle of $\mathcal{R}$. 
Definition 2.1. A sub- $\sigma$-algebra $\mathfrak{P}$ of $\mathfrak{B}_{X} \otimes \mathfrak{B}_{Y}$ is a generator for $\alpha$ if

$$
\bigvee_{x^{\prime} \in \mathcal{R}(x)} \alpha\left(x, x^{\prime}\right) \mathfrak{P}_{x^{\prime}}=\mathfrak{B}_{Y} \text { for a.e. } x
$$

We state also the dynamical analogue of this notion.

Definition 2.2. Let $\mathfrak{F}$ be a factor of an action $T=\left(T_{g}\right)_{g \in G}$ of a countable group $G$ on $(X, \mathfrak{B}, \mu)$. A sub- $\sigma$-algebra $\mathfrak{P}$ of $\mathfrak{B}_{X}$ is called an $\mathfrak{F}$-relative generator for $T$ if $\mathfrak{F} \vee \bigvee_{g \in G} T_{g} \mathfrak{P}=\mathfrak{B}_{X}$ for a.e. $x$.

Remark 2.3. It is easy to see that $\mathfrak{P}$ is a generator for $\alpha$ if and only if it is a $\left(\mathfrak{B}_{X} \otimes \mathfrak{N}_{Y}\right)$-relative generator for the $\alpha$-skew product extension of any action of a countable group generating $\mathcal{R}$.

The following statement is the orbital version of the relative Kolmogorov-Sinai theorem.

Lemma 2.4. Let $\mathcal{R}$ be hyperfinite. Given a generator $\mathfrak{P}$ for $\alpha$, we have $h(\alpha, \mathfrak{P})=$ $h(\alpha)$.

Proof. Suppose that the contrary holds: there exists a finite partition $Q$ of $X \times Y$ with $h(\alpha, Q)>h(\alpha, \mathfrak{P})$. By the Dye theorem [Dy] there is a transformation $\gamma$ of $X$ that generates $\mathcal{R}$. It is ergodic since so is $\mathcal{R}$. We deduce the following from Lemma 1.3 and the relative Pinsker formula for $\mathbb{Z}$-actions:

$$
\begin{aligned}
h(\alpha, \mathfrak{P}) & <h(\alpha, Q)=h\left(\gamma_{\alpha}, Q \mid \mathfrak{B}_{X} \otimes \mathfrak{N}_{Y}\right) \\
& \leq h\left(\gamma_{\alpha}, Q \vee \mathfrak{P} \mid \mathfrak{B}_{X} \otimes \mathfrak{N}_{Y}\right) \\
& =h\left(\gamma_{\alpha}, \mathfrak{P} \mid \mathfrak{B}_{X} \otimes \mathfrak{N}_{Y}\right)+h\left(\gamma_{\alpha}, Q \mid\left(\mathfrak{B}_{X} \otimes \mathfrak{N}_{Y}\right) \vee \bigvee_{i \in \mathbb{Z}} \gamma_{\alpha}^{i} \mathfrak{P}\right) \\
& =h(\alpha, \mathfrak{P})+0,
\end{aligned}
$$

since $\mathfrak{P}$ is a $\left(\mathfrak{B}_{X} \otimes \mathfrak{N}_{Y}\right)$-relative generator for $\gamma_{\alpha}$ (see Remark 2.3). Recall that $\gamma_{\alpha}$ stands for the $\alpha$-skew product extension of $\gamma$.

Definition 2.5. $\alpha$ is ergodic if so is the $\alpha$-skew product equivalence relation $\mathcal{R}(\alpha)$.

Clearly, if two cocycles are cohomologous or weakly equivalent and one of them is ergodic then so is the other one.

Now we state the orbital versions of the relative Krieger and Rokhlin theorems about generators.

Lemma 2.6. Let $\mathcal{R}$ be hyperfinite and $\alpha$ ergodic.

(i) If $h(\alpha)<\log k$ for an integer $k \geq 2$ then there exists a finite generator $P$ for $\alpha$ with $\# P=k$.

(ii) If $h(\alpha)=\infty$ then there exists a countable generator for $\alpha$.

Proof. (i) Let $\gamma$ be a zero entropy transformation that generates $\mathcal{R}$. We deduce from the Abramov-Rokhlin entropy addition formula [AR] and Lemma 1.3 that

$$
h\left(\gamma_{\alpha}\right)=h(\gamma)+h\left(\gamma_{\alpha} \mid \mathfrak{B}_{X} \otimes \mathfrak{N}_{Y}\right)=h(\alpha)
$$


Since $\alpha$ is ergodic and the transformation $\gamma_{\alpha}$ generates $\mathcal{R}(\alpha)$, we deduce that $\gamma_{\alpha}$ is ergodic. Then by the Krieger theorem $[\mathrm{Kr}]$, there exists an (absolute) generator $P$ for $\gamma_{\alpha}$ with $\# P=k[\mathrm{Kr}]$. Clearly, $P$ is a $\left(\mathfrak{B}_{X} \otimes \mathfrak{N}_{Y}\right)$-relative generator for $\gamma_{\alpha}$ as well. It remains to apply Remark 2.3 .

(ii) can be demonstrated in a similar way: just apply the Rokhlin theorem about countable generators [Rok] instead of the Krieger theorem.

From Lemmas 2.4 and 2.6 we deduce respectively the relative Kolmogorov-Sinai, Krieger and Rokhlin theorems for amenable group actions (see Remark 2.3).

Theorem 2.7. Let $T$ be an ergodic action of a countable amenable group and $\mathfrak{F} a$ class-bijective factor of $T$.

(i) If $P$ is a finite $\mathfrak{F}$-relative generator for $T$ then $h(T, P \mid \mathfrak{F})=h(T \mid \mathfrak{F})$.

(ii) If $h(T \mid \mathfrak{F})<\log k$ then there is a finite $\mathfrak{F}$-relative generator $P$ for $T$ with $\# P=k$.

(iii) If $h(T \mid \mathfrak{F})=\infty$ then there is a countable $\mathfrak{F}$-relative generator for $T$.

\section{Bernoullian COCYCles AND Relatively BeRnoullian Group aCtions}

In this section we introduce the concept of Bernoullicity for cocycles. We discuss how it is linked with the concept of relative Bernoullicity for countable group actions. Such actions split into the product of the original factor and a Bernoullian one. We show that a cocycle is Bernoullian if and only if the corresponding skew product extension of every group action generating the underlying equivalence relation is relatively Bernoullian. This holds in the general setting of countable group actions and discrete equivalence relations. Then we study the case of amenable groups and hyperfinite relations in more detail. We prove the "uniqueness" theorem for Bernoullian cocycles of equal entropy and demonstrate the existence of Bernoullian generators with prescribed distributions for Bernoullian cocycles. As a corollary, we deduce similar results for relatively Bernoullian amenable group actions.

Definition 3.1. We say that a finite partition $P$ of $X \times Y$ is Bernoullian for $\alpha$ if $h(\alpha, P)=H(P)$. A sub- $\sigma$-algebra $\mathfrak{P}$ of $\mathfrak{B}_{X} \otimes \mathfrak{B}_{Y}$ is Bernoullian for $\alpha$ if so is every finite $\mathfrak{P}$-measurable partition of $X \times Y$. We call $\alpha$ Bernoullian if there exists a Bernoullian generator for it.

Clearly, if two cocycles are cohomologous or weakly equivalent and one of them is Bernoullian then so is the other one.

Remark 3.2. It follows from Lemma 2.4 that if $\mathcal{R}$ is hyperfinite then a finite generator $P$ is Bernoullian if and only if $H(P)=h(\alpha)$.

Proposition 3.3. A sub- $\sigma$-algebra $\mathfrak{P}$ is Bernoullian for $\alpha$ if and only if it is independent of $\mathfrak{B}_{X} \otimes \mathfrak{N}_{Y}$ and the sub- $\sigma$-algebras $\alpha\left(x, x^{\prime}\right) \mathfrak{P}_{x^{\prime}}$ of $\mathfrak{B}_{Y}, x^{\prime} \in \mathcal{R}(x)$, are independent for a.e. $x \in X$.

Proof. It is enough to consider only $\mathfrak{P}$ finite. The general case can be deduced from this via the standard approximation of $\mathfrak{P}$ by an increasing sequence of finite sub- $\sigma$-algebras. 
Thus suppose that $P$ is a finite Bernoullian partition for $\alpha$. Given a type $I$ subrelation $\mathcal{S} \in \mathcal{R}$, we have

$$
\begin{aligned}
H(P) & =h(\alpha, P) \leq h(\mathcal{S}, \alpha, P) \\
& =\int \frac{1}{\# \mathcal{S}(x)} H\left(\bigvee_{x^{\prime} \in \mathcal{S}(x)} \alpha\left(x, x^{\prime}\right) P_{x^{\prime}}\right) d \mu(x) \\
& \leq \int \frac{1}{\# \mathcal{S}(x)} \sum_{x^{\prime} \in \mathcal{S}(x)} H\left(P_{x^{\prime}}\right) d \mu(x) \\
& =\int E\left(H\left(P_{x}\right) \mid \mathcal{S}\right) d \mu(x) \\
& =\int H\left(P_{x}\right) d \mu(x)=H\left(P \mid \mathfrak{B}_{X} \otimes \mathfrak{N}_{Y}\right) \leq H(P),
\end{aligned}
$$

where $E(. \mid \mathcal{S})$ stands for the conditional expectation relative to the $\sigma$-algebra of $\mathcal{S}$-invariant subsets. It follows that the finite $Y$-partitions $\alpha\left(x, x^{\prime}\right) P_{x^{\prime}}, x^{\prime} \in \mathcal{S}(x)$, are independent for a.e. $x$. Since by $[\mathrm{FM}]$ every discrete equivalence relation is the union of a countable family of type $I$ subrelations, the partitions $\alpha\left(x, x^{\prime}\right) P_{x^{\prime}}$, $x^{\prime} \in \mathcal{R}(x)$, are independent for a.e. $x \in X$. From (3-1) we deduce as well that $H\left(P \mid \mathfrak{B}_{X} \otimes \mathfrak{N}_{Y}\right)=H(P)$ which yields $P \perp\left(\mathfrak{B}_{X} \otimes \mathfrak{N}_{Y}\right)$.

To prove the opposite claim it suffices to notice that $h(S, \alpha, P)=H(P)$ for every type $I$ subrelation $\mathcal{S} \subset \mathcal{R}$. This fact follows straightforward from our assumptions on $P$ and (3-1) (now with $=$ 's instead of $\leq$ 's).

Example 3.4. Let $G$ be a countable (not necessarily amenable) group, $T=$ $\left(T_{g}\right)_{g \in G}$ a free $G$-action generating $\mathcal{R}$ and $L=\left(L_{g}\right)_{g \in G}$ a Bernoullian action of $G$ on $(Y, \nu)$. We set

$$
\beta\left(T_{g} x, x\right)=L_{g}, \quad g \in G, x \in X
$$

Then $\beta$ is a Bernoullian cocycle and if a sub- $\sigma$-algebra $\mathfrak{Q} \subset \mathfrak{B}_{Y}$ is a Bernoullian generator for $L$ then $\mathfrak{N}_{X} \otimes \mathfrak{Q}$ is a Bernoullian generator for $\beta$.

Now we state the dynamical counterparts of the Bernoullicity concepts from Definition 3.1.

Definition 3.5. Let $\mathfrak{F}$ be a factor of an action $T=\left(T_{g}\right)_{g \in G}$ of a countable group $G$ on $(X, \mathfrak{B}, \mu)$. A finite partition $P$ of $X$ is called $\mathfrak{F}$-relatively Bernoullian if $h(T, P \mid \mathfrak{F})=H(P)$. A sub- $\sigma$-algebra $\mathfrak{P}$ of $\mathfrak{B}_{X}$ is $\mathfrak{F}$-relatively Bernoullian if so is every finite $\mathfrak{P}$-measurable partition of $X$.

If there exists an $\mathfrak{F}$-relatively Bernoullian generator for $T$ then $T$ is called $\mathfrak{F}$ relatively Bernoullian.

Remark 3.6. It follows from (1-1) that $T$ is $\mathfrak{F}$-relatively Bernoullian if and only if there exists a sub- $\sigma$-algebra $\mathfrak{P}$ of $\mathfrak{B}_{X}$ with the following properties (cf. Proposition 3.3):

(i) $\mathfrak{P}$ is independent under $T$, i.e. the sub- $\sigma$-algebras $T_{g} \mathfrak{P}, g \in G$, are independent,

(ii) $\mathfrak{F} \perp \bigvee_{g \in G} T_{g} \mathfrak{P}$ and

(iii) $\mathfrak{F} \vee \bigvee_{g \in G} T_{g} \mathfrak{P}=\mathfrak{B}_{X}$. 
Hence there exists a measure space isomorphism

$$
\tau:\left(X, \mathfrak{B}_{X}, \mu\right) \rightarrow(X, \mathfrak{F}, \mu \uparrow \mathfrak{F}) \times\left(Y, \mathfrak{B}_{Y}, \nu\right)
$$

such that the following are satisfied:

(i) there are a Bernoullian $G$-action $L$ on $(Y, \nu)$ and a Bernoullian generator $\mathfrak{Q} \subset \mathfrak{B}_{Y}$ for $L$ with $\tau(\mathfrak{P})=\mathfrak{N}_{X} \otimes \mathfrak{Q}$,

(ii) $\tau(A)=A$ for every subset $A \in \mathfrak{F}$,

(iii) $\tau T \tau^{-1}=(T \nmid \mathfrak{F}) \times L$.

To put it in other way, $T$ splits into the Cartesian product of the factor action $T \uparrow \mathfrak{F}$ and another (Bernoullian) factor of $T$ generated by $\mathfrak{P}$. Clearly,

$$
h(T, \mathfrak{P} \mid \mathfrak{F})=h(L, \mathfrak{Q})=H(\mathfrak{Q}) .
$$

Remark 3.7. Notice that if $T$ is $\mathfrak{F}$-relatively Bernoullian then it is free (provided that $\mathfrak{F} \neq \mathfrak{B}_{X}$ ).

We explain the interplay between the orbital and dynamical Bernoullicity in the next statement. Notice that in the particular case of amenable group actions (and hyperfinite $\mathcal{R}$ ) it follows easily from Lemma 1.3.

Proposition 3.8. Let $\mathcal{R}$ be generated by a free action $T$ of a countable group $G$. Then a finite partition $P$ of $X \times Y$ is Bernoullian for $\alpha$ if and only if it is $\left(\mathfrak{B}_{X} \otimes \mathfrak{N}_{Y}\right)$ relatively Bernoullian for the $\alpha$-skew product extension $T^{\alpha}$ of T. Moreover, $\alpha$ is Bernoullian if and only if $T^{\alpha}$ is $\left(\mathfrak{B}_{X} \otimes \mathfrak{N}_{Y}\right)$-relatively Bernoullian.

Proof. $(\Longleftarrow)$ Let $F$ be a finite subset in $G$. Since $P$ is $\left(\mathfrak{B}_{X} \otimes \mathfrak{N}_{Y}\right)$-relatively Bernoullian for $T^{\alpha}$, we have

$$
\begin{aligned}
H(P) & \leq \frac{1}{\# F} H\left(\bigvee_{g \in F} T_{g}^{\alpha} P \mid \mathfrak{B}_{X} \otimes \mathfrak{N}_{Y}\right) \\
& =\int_{X} \frac{1}{\# F} H\left(\bigvee_{g \in F} \alpha\left(x, T_{g} x\right) P_{T_{g} x}\right) d \mu(x) \\
& \leq \int_{X} \frac{1}{\# F} \sum_{g \in F} H\left(P_{T_{g} x}\right) d \mu(x) \\
& =H\left(P \mid \mathfrak{B}_{X} \otimes \mathfrak{N}_{Y}\right)=H(P) .
\end{aligned}
$$

It follows that the partitions $P_{T_{g} x}, g \in F$, are independent for a.a. $x \in X$. Since $T$ generates $\mathcal{R}$, it follows that the partitions $P_{x^{\prime}}, x^{\prime} \in \mathcal{R}(x)$, are independent for a.a. $x$. Hence $P$ is Bernoullian for $\alpha$.

$(\Longrightarrow)$ Given a finite subset $F \subset G$, let $X=\bigcup_{i} X_{i}$ be a countable partition of $X$ such that for each $i$ the subsets $T_{g} X_{i}, g \in F$, are disjoint. Existence of such a partition is a simple corollary from the freeness of $T$. For the proof, see for instance [OrW, p.57] - amenability of $G$ is used nowhere in that proof. Define an equivalence relation $\mathcal{S}_{i}$ on $X$ by setting $x \sim_{\mathcal{S}_{i}} x^{\prime}$ if $x=x^{\prime}$ or $x, x^{\prime} \in\left\{T_{g} z \mid g \in F\right\}$ for some $z \in X_{i}$. Clearly, $\mathcal{S}_{i}$ is a type $I$ subrelation of $\mathcal{R}$. It is easy to see that

$$
h\left(\mathcal{S}_{i}, \alpha, P\right)=\int_{X \backslash \bigcup_{g \in F} T_{g} X_{i}} H\left(P_{x}\right) d \mu(x)+\int_{X_{i}} H\left(\bigvee_{g \in F} \alpha\left(x, T_{g} x\right) P_{T_{g} x}\right) d \mu(x) .
$$


On the other hand, $h\left(\mathcal{S}_{i}, \alpha, P\right)=H\left(P \mid \mathfrak{B}_{X} \otimes \mathfrak{N}_{Y}\right)$ since $P$ is Bernoullian for $\alpha$. It follows that

$$
\frac{1}{\# F} \int_{X_{i}} H\left(\bigvee_{g \in F} \alpha\left(x, T_{g} x\right) P_{T_{g} x}\right) d \mu(x)=\frac{1}{\# F} \int_{\bigcup_{g \in F} T_{g} X_{i}} H\left(P_{x}\right) d \mu(x)
$$

Of course,

$$
\sum_{i} 1_{\cup_{g \in F} T_{g} X_{i}}=\sum_{i} \sum_{g \in F} 1_{T_{g} X_{i}}=\sum_{g \in F} 1_{X}=\# F \text { a.e.. }
$$

Hence summing (3-3) in $i$ we obtain

$$
\frac{1}{\# F} H\left(\bigvee_{g \in F} T_{g}^{\alpha} P \mid \mathfrak{B}_{X} \otimes \mathfrak{N}_{Y}\right)=H\left(P \mid \mathfrak{B}_{X} \otimes \mathfrak{N}_{Y}\right)=H(P),
$$

as desired.

The second claim of the proposition follows from the first one and Remark 2.3.

Since the Cartesian product of two Bernoullian $G$-actions is a Bernoullian $G$ action, we conclude that Bernoullicity is preserved under extending by Bernoullian cocycles (like the CPE-property of amenable group actions is preserved under extending by CPE-cocycles):

Corollary 3.9. Let $\mathcal{R}$ be generated by a Bernoullian action $T$ of a countable group and $\alpha$ a Bernoullian cocycle of $\mathcal{R}$. Then the $\alpha$-skew product extension $T^{\alpha}$ of $T$ is also Bernoullian. Moreover, if $\mathfrak{Q}$ is an independent generator for $T$ and $\mathfrak{P}$ a Bernoullian generator for $\alpha$ then $\left(\mathfrak{Q} \otimes \mathfrak{N}_{Y}\right) \vee \mathfrak{P}$ is a Bernoullian generator for $T^{\alpha}$.

The next assertion about "straightening" of cocycles follows from Proposition 3.8 and Remarks 3.6 and 3.2.

Corollary 3.10. Let $\mathcal{R}$ be generated by a free $G$-action and $\alpha$ a Bernoullian cocycle of $\mathcal{R}$. Then there exists a Bernoulian $G$-action $L$ on $(Y, \nu)$ such that $\alpha$ is cohomologous to a cocycle $\beta$ given by (3-2). Moreover, if $\mathcal{R}$ is hyperfinite then $h(\alpha)=h(L)$.

In particular, we conclude from this that every Bernoullian cocycle is ergodic. Furthermore, if $\mathcal{R}$ is hyperfinite then $\alpha$ is CPE. Actually, it is easy to verify that the property of cocycles to be CPE is a cohomology invariant [Da3]. Since $\mathcal{R}$ is generated by a single transformation $\gamma$, it follows from Corollary 3.10 and the last assertion of Lemma 1.3 that $\alpha$ is CPE whenever the Cartesian product of $\gamma$ with some Bernoullian transformation of $Y$ is $\left(\mathfrak{B}_{X} \otimes \mathfrak{N}_{Y}\right)$-relatively CPE. But the last claim is a well known classical fact.

It is easy to verify that the entropy of cocycles is a cohomology invariant (see [Da3]). We show now that this invariant is complete in the class of Bernoullian cocycles of hyperfinite equivalence relations.

Theorem 3.11. Let $\mathcal{R}$ be hyperfinite and $\alpha, \beta: \mathcal{R} \rightarrow \operatorname{Aut}(Y, \nu)$ two Bernoullian cocycles. They are cohomologous if and only if $h(\alpha)=h(\beta)$.

Proof. Let $\gamma$ be a transformation that generates $\mathcal{R}$. By Corollary 3.10, there exist cocycles $\alpha^{\prime}$ and $\beta^{\prime}$ of $\mathcal{R}$ cohomologous to $\alpha$ and $\beta$ respectively such that

$$
\alpha^{\prime}(\gamma x, x)=\theta_{1}, \quad \beta^{\prime}(\gamma x, x)=\theta_{2},
$$


where $\theta_{1}$ and $\theta_{2}$ are two Bernoullian transformations. Moreover,

$$
h\left(\theta_{1}\right)=h(\alpha)=h(\beta)=h\left(\theta_{2}\right) .
$$

Hence by the Ornstein isomorphism theorem (see [Or1] and [Or2]), there exists a transformation $\delta \in \operatorname{Aut}(Y, \nu)$ such that $\theta_{1}=\delta \theta_{2} \delta^{-1}$. It follows that $\alpha^{\prime}$ and $\beta^{\prime}$ (and hence $\alpha$ and $\beta$ ) are cohomologous. Actually, put $\phi(x):=\delta$ for a.e. $x \in X$. Then $\phi$ is a "transfer function" from $\alpha^{\prime}$ to $\beta^{\prime}$.

Remark 3.12. Let $\mathcal{R}$ be hyperfinite and $\alpha$ Bernoullian. It is easy to verify that for any transformation $\theta \in N[\mathcal{R}]$, the cocycle $\alpha \circ \theta$ (see $\S 2$ for the definition) is also Bernoullian. Moreover, $h(\alpha \circ \theta)=h(\alpha)$ [Da3]. Hence $\alpha \circ \theta$ is cohomologous to $\alpha$ according to Theorem 3.11. Thus the "symmetry group"

$$
D(\mathcal{R}, \alpha):=\{\theta \in N[\mathcal{R}] \mid \alpha \circ \theta \text { is cohomologous to } \alpha\}
$$

of $\alpha$ is the entire normalizer $N[\mathcal{R}]$, i.e. the largest possible. This is in a sharp contrast with the cocycles taking values in compact groups (and, in fact with all regular cocycles [Da2]): if the symmetry group of such a cocycle is $N[\mathcal{R}]$ then this cocycle is a coboundary [Da1], [DG]. We conjecture that if $\mathcal{R}$ is hyperfinite, $\alpha$ ergodic and $D(\mathcal{R}, \alpha)=N[\mathcal{R}]$ then $\alpha$ is Bernoullian.

Next we prove that a relatively Bernoullian amenable group action has a relatively Bernoullian generator with any prescribed distribution (of fixed entropy). To do this we first establish a similar result for Bernoullian cocycles. Recall first that a $k$-element distribution $I$ is a probability vector $\left(I_{1}, \ldots, I_{k}\right)$. The entropy of $I$ is $H(I):=-\sum_{n=1}^{k} I_{n} \log I_{n}$. From now on we assume that a partition, say $P$ of $\left(X, \mathfrak{B}_{X}, \mu\right)$, is ordered, i.e. $P=\left(P_{1}, \ldots, P_{k}\right)$ with a fixed order. The associated distribution $\left(\mu\left(P_{1}\right), \ldots, \mu\left(P_{k}\right)\right)$ is denoted by dist $P$. In a similar way we can define $H(I)$ and dist $P$ for a countable distribution $I=\left(I_{1}, I_{2}, \ldots\right)$ and countable ordered partition $P=\left(P_{1}, P_{2}, \ldots\right)$ respectively.

Proposition 3.13. Let $T$ be a free ergodic action of an amenable group $G$ and $\mathfrak{F}$ a class-bijective factor of $T$. If $T$ is $\mathfrak{F}$-relatively Bernoullian then for each finite or countable distribution I with $H(I)=h(T \mid \mathfrak{F})$ there exists a finite or countable $\mathfrak{F}$-relatively Bernoullian generator $P$ of $T$ with dist $P=\operatorname{dist} I$. In particular, if $h(T \mid \mathfrak{F})=\log k$ then there is a $k$-element $\mathfrak{F}$-relatively Bernoullian generator of $T$. Conversely, if $T$ admits a k-element generator $P$ with $h(T \mid \mathfrak{F})=\log k$ then $P$ is $\mathfrak{F}$-relatively Bernoullian.

In view of Proposition 3.8, Remarks 2.3 and 3.2, to settle Proposition 3.13 it suffices to prove

Lemma 3.14. Let $\mathcal{R}$ be hyperfinite and $\alpha$ Bernoullian. Given a finite or countable distribution I with $h(\alpha)=H(I)$, there is a finite or countable Bernoullian generator $P$ for $\alpha$ with dist $P=\operatorname{dist} I$. In particular, if $h(\alpha)=\log k$ then there exists a Bernoullian k-element generator. Conversely, if $\alpha$ admits a $k$-element generator $P$ and $h(\alpha)=\log k$, then $\alpha$ is Bernoullian.

Proof. The first assertion follows from Theorem 3.11 and Example 3.4. The second one is obvious. We deduce the last assertion from the inequality

$$
\log k=h(\alpha, P) \leq H(P) \leq \log k .
$$




\section{Factors, extensions and Relative Bernoulli theOry}

In this section we use the orbital approach to extend (a part of) the Thouvenot relative isomorphism theory [Th1], [Th2] (see also [Li] for the case of infinite entropy) and several results from [Ho] to amenable group actions. Among them are relative Sinai theorem on Bernoullian factors, Thouvenot theorem about relative Bernoullicity of a factor of a relatively Bernoullian transformation, Thouvenot theorem about factors of transformations with the strong Pinsker property, Hoffman's uncountable family of non-relatively-isomorphic relatively CPE transformations with the same relative entropy, etc. To achieve this we introduce a concept of factor for cocycles of measured equivalence relations. This concept correspond exactly to that of relative factor for group actions (i.e. a factor which contains another-fixed-one). Hence relative factors are invariant under the factor orbit equivalence. The same is true for the subclass of relatively Bernoullian factors, i.e. factors with a complementary Bernoullian factor. This enable us to deduce the desired results from their classical counterparts.

From now on we will assume that $\mathcal{R}$ is hyperfinite and $G$ amenable.

Definition 4.1. A sub- $\sigma$-algebra $\mathfrak{F}=\left(\mathfrak{F}_{x}\right)_{x \in X} \subset \mathfrak{B}_{X} \otimes \mathfrak{B}_{Y}$ is called a factor of $\alpha$ if $\mathfrak{F} \supset \mathfrak{B}_{X} \otimes \mathfrak{N}_{Y}$ and $\mathfrak{F}_{x}=\alpha\left(x, x^{\prime}\right) \mathfrak{F}_{x^{\prime}}$ at all $\left(x, x^{\prime}\right) \in \mathcal{R} \cap(B \times B)$ for a $\mu$ conull subset $B$. (Recall that $\left(\mathfrak{F}_{x}\right)_{x \in X}$ stands for the associated measurable field of sub- $\sigma$-algebras of $\mathfrak{B}_{Y}$-see $\S 1$.)

Since $\mathfrak{F}$ contains $\mathfrak{B}_{X} \otimes \mathfrak{N}_{Y}$, the associated measurable field $\left(\mathfrak{F}_{x}\right)_{x \in X}$ determines $\mathfrak{F}$ uniquely, i.e. if $\mathfrak{F}$ and $\mathfrak{F}^{\prime}$ are two factors of $\alpha$ with $\mathfrak{F}_{x}=\mathfrak{F}_{x}^{\prime}$ a.e. then $\mathfrak{F}=\mathfrak{F}^{\prime}$.

\section{Definition 4.2.}

(i) A factor $\mathfrak{F}=\left(\mathfrak{F}_{x}\right)_{x \in X}$ of $\alpha$ is called Bernoullian if there exists a Bernoullian sub- $\sigma$-algebra $\mathfrak{P} \subset \mathfrak{B}_{X} \otimes \mathfrak{B}_{Y}$ such that $\bigvee_{x^{\prime} \in \mathcal{R}(x)} \alpha\left(x, x^{\prime}\right) \mathfrak{P}_{x}=\mathfrak{F}_{x}$ for a.a. $x$.

(ii) A factor of $\alpha$ is called prime if it has no proper factors except for $\mathfrak{B}_{X} \otimes \mathfrak{N}_{Y}$.

Remark 4.3 .

(i) Let a sub- $\sigma$-algebra $\mathfrak{F}$ of $\mathfrak{B}_{X} \otimes \mathfrak{B}_{Y}$ contains $\mathfrak{B}_{X} \otimes \mathfrak{N}_{Y}$. Then $\mathfrak{F}$ is a factor of $\alpha$ if and only if $\mathfrak{F}$ is a factor of the $\alpha$-skew product extension $T^{\alpha}$ of any countable group action $T$ generating $\mathcal{R}$.

(ii) A factor $\mathfrak{F}$ is Bernoullian for $\alpha$ if and only if it is $\left(\mathfrak{B}_{X} \otimes \mathfrak{N}_{Y}\right)$-relatively Bernoullian for every such a $T^{\alpha}$.

(iii) A factor $\mathfrak{F}$ of $\alpha$ is prime if it $\left(\mathfrak{B}_{X} \otimes \mathfrak{N}_{Y}\right)$-relatively prime for every $T^{\alpha}$. Recall that a $G$-action $T$ is called relatively prime with respect to a factor $\mathfrak{E}$ if $T$ has no proper factors containing $\mathfrak{E}$ except for $\mathfrak{E}$ itself.

(iv) Every sub- $\sigma$-algebra $\mathfrak{P} \subset \mathfrak{B}_{X} \otimes \mathfrak{B}_{Y}$ is a generator of some factor of $\alpha$. This factor is determined uniquely by $\mathfrak{P}$. Actually, consider a measurable field $X \ni x \mapsto \bigvee_{x^{\prime} \in \mathcal{R}(x)} \alpha\left(x, x^{\prime}\right) \mathfrak{P}_{x^{\prime}}$ of sub- $\sigma$-algebras of $\mathfrak{B}_{Y}$. Clearly, this measurable field determines uniquely a factor of $\alpha$ (see the remark just after Definition 4.1).

We are going to establish the relative Sinai theorem (about Bernoullian factors) for amenable group actions and - as a by-product - for cocycles of hyperfinite equivalence relations. Recall first that the distance between two $k$-element distributions $I$ and $I^{\prime}$ is $\left|I-I^{\prime}\right|:=\sum_{n=1}^{k}\left|I_{n}-I_{n}^{\prime}\right|$ and the $L^{1}$-distance between two $k$-element partitions $P$ and $P^{\prime}$ is $\left\|P-P^{\prime}\right\|_{1}:=\sum_{n=1}^{k} \mu\left(P_{n} \triangle P_{n}^{\prime}\right)$. 
Now we state the Ornstein's version of the relative Sinai theorem for $\mathbb{Z}$-actions (see [Or2, Lemma 5] and [Th1, Proposition 2']) in the following (a bit different but equivalent) form:

Lemma 4.4. Let $\gamma$ be an ergodic transformation of $X, \mathfrak{F}$ is a factor of $\gamma$ with $h(\gamma, \mathfrak{F})<\infty$ and $I$ a k-element distribution with $H(I) \leq h(\gamma \mid \mathfrak{F})$. Then for each $\epsilon>0$ there is $\delta>0$ such that for every $k$-element partition $P^{\prime}$ with

(i) $\left|\operatorname{dist} P^{\prime}-I\right|<\delta$ and

(ii) $\left|h\left(\gamma, P^{\prime} \mid \mathfrak{F}\right)-H(I)\right|<\delta$

there exists a $k$-element $\mathfrak{F}$-relatively Bernoullian partition $P$ such that

(iii) $\left\|P^{\prime}-P\right\|_{1}<\epsilon$ and

(iv) $\operatorname{dist} P=I$.

Proposition 4.5. let $T$ be a free ergodic action of $G, \mathfrak{F}$ a class-bijective factor of $T$ and $I$ a k-element distribution with $H(I) \leq h(T \mid \mathfrak{F})$. Then for each $\epsilon>0$ there is $\delta>0$ such that for every $k$-element partition $P^{\prime}$ with

(i) $\left|\operatorname{dist} P^{\prime}-I\right|<\delta$ and

(ii) $\left|h\left(T, P^{\prime} \mid \mathfrak{F}\right)-H(I)\right|<\delta$

there exists a $k$-element $\mathfrak{F}$-relatively Bernoullian partition $P$ such that

(iii) $\left\|P^{\prime}-P\right\|_{1}<\epsilon$ and

(iv) $\operatorname{dist} P=I$.

Proof. We first restate the claim of the proposition into the language of discrete equivalence relations and their cocycles as follows:

Let $\alpha: \mathcal{R} \rightarrow \operatorname{Aut}(Y, \nu)$ be an ergodic cocycle of $\mathcal{R}$. Given a $k$-element distribution $I$ with $H(I) \leq h(\alpha)$ and $\epsilon>0$ there is $\delta>0$ such that for every $k$-element partition $P^{\prime}$ of $X \times Y$ with

(a) $\left|\operatorname{dist} P^{\prime}-I\right|<\delta$ and

(b) $\left|h\left(\alpha, P^{\prime}\right)-H(I)\right|<\delta$

there exists a $k$-element Bernoullian partition $P$ of $X \times Y$ such that

(c) $\left\|P^{\prime}-P\right\|_{1}<\epsilon$ and

(d) $\operatorname{dist} P=I$.

But this is surely true. Actually, just put $\mathfrak{F}:=\mathfrak{B}_{X} \otimes \mathfrak{N}_{Y}$ and $\gamma:=\theta_{\alpha}$ for a transformation $\theta$ generating $\mathcal{R}$ and apply Lemma 4.4 and Remark 4.3(i, ii).

To complete the proof, notice that every group action having a class-bijective factor can be represented as a skew product extension over this factor and that the orbit equivalence relation of $T$ is hyperfinite [CFW]. It remains to apply again Remark 4.3(i, ii) but this time in the "opposite direction".

In particular, the following statement holds (and that is exactly what we need for applications in the further sections):

Corollary 4.6. Given a class-bijective factor $\mathfrak{F}$ of a free ergodic action $T$ of $G$ and a finite distribution $I$ such that $H(I) \leq h(T \mid \mathfrak{F})$, there exists a Bernoullian factor $\mathfrak{E} \perp \mathfrak{F}$ having a finite Bernoullian generator $Q$ with $\operatorname{dist} Q=I$. The same is true for a countable distribution $I$, and if $H(I)=\infty$ we can claim in addition that $h\left(T \mid \mathfrak{F} \vee \bigvee_{g \in G} T_{g} Q\right)=0$

Proof. The first assertion follows immediatly from Proposition 4.5. To prove the second one we consider separately two cases: 
(A) $H(I)<\infty$. Take a finite distribution $I^{\prime}$ with $H\left(I^{\prime}\right)=H(I)$, apply the first assertion of the corollary to $I^{\prime}$ and - to conclude - make use of Proposition 3.13.

(B) $H(I)=\infty$ and hence $h(T \mid \mathfrak{F})=\infty$. By Theorem 2.7(iii), there exists a countable $\mathfrak{F}$-relative generator $P=\left(P_{1}, P_{2}, \ldots\right)$ for $T$. Let $\mathfrak{F}_{n}$ stand for the $\mathfrak{F}$-relative factor of $T$ generated by the finite partition $\left(P_{1}, \ldots, P_{n}, X \backslash \bigcup_{i=1}^{n} P_{i}\right)$, $n=1,2 \ldots$ and let $\mathfrak{F}_{0}:=\mathfrak{F}$. By the first claim of the corollary, there exists a Bernoullian factor $\mathfrak{E}_{n} \subset \mathfrak{F}_{n}$ such that $\mathfrak{E}_{n} \perp \mathfrak{F}_{n-1}$ and

$$
h\left(T, \mathfrak{E}_{n} \mid \mathfrak{F}_{n-1}\right)=h\left(T, \mathfrak{F}_{n} \mid \mathfrak{F}_{n-1}\right) .
$$

Notice that $h\left(T, \mathfrak{F}_{n} \mid \mathfrak{F}_{n-1}\right) \leq h\left(T, \mathfrak{F}_{n} \mid \mathfrak{F}\right) \leq \log (n+1)$. Since $\mathfrak{E}_{n}, n=1,2, \ldots$, are pairwise independent, the $T$-factor $\mathfrak{E}:=\bigvee_{n=1}^{\infty} \mathfrak{E}_{n}$ is Bernoullian. Remark also that $\mathfrak{E}_{n} \perp \mathfrak{F}$ for all $n>0$ and hence $\mathfrak{E} \perp \mathfrak{F}$. By Proposition 3.13, we can find a countable Bernoullian partition $Q$ of $X$ with dist $Q=I$ and $\bigvee_{g \in G} T_{g} Q \vee \mathfrak{F}=\mathfrak{E} \vee \mathfrak{F}$ It follows from (4-1) that $h\left(T, \mathfrak{F}_{n} \mid \mathfrak{F}_{n-1} \vee \mathfrak{E}_{n}\right)=0$ and hence

$$
\begin{aligned}
h\left(T, \mathfrak{F}_{n} \mid \mathfrak{F} \vee \mathfrak{E}\right) & \leq h\left(T, \mathfrak{F}_{n} \mid \mathfrak{F} \vee \mathfrak{E}_{1} \vee \cdots \vee \mathfrak{E}_{n}\right) \\
& =h\left(T, \mathfrak{F}_{n-1} \mid \mathfrak{F} \vee \mathfrak{E}_{1} \vee \cdots \vee \mathfrak{E}_{n}\right)+h\left(T, \mathfrak{F}_{n} \mid \mathfrak{E}_{1} \vee \cdots \vee \mathfrak{E}_{n} \vee \mathfrak{F}_{n-1}\right) \\
& \leq h\left(T, \mathfrak{F}_{n-1} \mid \mathfrak{F} \vee \mathfrak{E}_{1} \vee \cdots \vee \mathfrak{E}_{n-1}\right) \\
& \cdots \\
& \leq h(T, \mathfrak{F} \mid \mathfrak{F})=0 .
\end{aligned}
$$

Since $\mathfrak{F}_{1} \subset \mathfrak{F}_{2} \subset \cdots$ and $\bigvee_{n=1}^{\infty} \mathfrak{F}_{n}=\mathfrak{B}_{X}$, we obtain $h(T \mid \mathfrak{F} \vee \mathfrak{E})=0$, as desired.

Remark that now we do not need the condition $h(T, \mathfrak{F})<\infty$ from [Th1, Proposition 2'].

Our next objective is to extend the main results from [Th1] and [Th2] to the general setting of amenable group actions (see Theorems 4.9 and 4.12 ). We recall first

Lemma 4.7 ([Th1, Proposition 5] and [Li, Appendix]). Let $\gamma$ be an ergodic transformation, $\mathfrak{F} \subset \mathfrak{H}$ two factors of it. If $\gamma$ is $\mathfrak{F}$-relatively Bernoullian then so is $\gamma\lceil\mathfrak{H}$.

Lemma 4.8 [Th2, Lemma 1]. Let $\gamma$ be an ergodic transformation of $\left(Z, \mathfrak{B}_{Z}, \kappa\right)$ with finite entropy and $\mathfrak{F}$ and $\mathfrak{H}$ two factors of $\gamma$ such that the following are satisfied:

(i) $\mathfrak{F} \vee \mathfrak{H}=\mathfrak{B}_{Z}$,

(ii) $\gamma$ is $\mathfrak{H}$-relatively Bernoullian,

(iii) $\mathfrak{F} \perp_{\mathfrak{F} \cap \mathfrak{H}} \mathfrak{H}$.

Then $\mathfrak{F}$ is $(\mathfrak{F} \cap \mathfrak{H})$-relatively Bernoullian.

We extend these results (provided that the factors are class-bijective) as follows.

Theorem 4.9. Let $T$ be an ergodic action of $G$ and $\mathfrak{F} \subset \mathfrak{H}$ two factors of $T$. If $\mathfrak{F}$ is class-bijective and $T$ is $\mathfrak{F}$-relatively Bernoullian then so is $T \uparrow \mathfrak{H}$.

Proof. We first establish an orbital version of this proposition. Namely, let $\alpha$ be a Bernoullian cocycle of $\mathcal{R}$. Then every factor $\mathfrak{F}$ of $\alpha$ is Bernoullian.

Actually, let $\gamma$ be a transformation of $X$ that generates $\mathcal{R}$. By Proposition 3.8, the $\alpha$-skew product extension $\gamma_{\alpha}$ of $\gamma$ is $\left(\mathfrak{B}_{X} \otimes \mathfrak{N}_{Y}\right)$-relatively Bernoullian. Since 
$\mathfrak{F} \supset \mathfrak{B}_{X} \otimes \mathfrak{N}_{Y}$, we deduce from Lemma 4.7 that $\gamma_{\alpha}\left\lceil\mathfrak{F}\right.$ is $\left(\mathfrak{B}_{X} \otimes \mathfrak{N}_{Y}\right)$-relatively Bernoullian. Hence $\mathfrak{F}$ is Bernoullian for $\alpha$ (see Remark 4.3(ii)), as claimed.

Now to deduce the statement of the proposition from this claim do exactly like at the final step of the proof of Proposition 4.5.

Lemma 4.10. Let $T$ be an ergodic free action of $G$ on $\left(X, \mathfrak{B}_{X}, \mu\right)$ and $\mathfrak{F}$ and $\mathfrak{H}$ two factors of $T$ such that the following are satisfied:

(i) $\mathfrak{F} \vee \mathfrak{H}=\mathfrak{B}_{X}$,

(ii) $h(T, \mathfrak{H})<\infty$,

(iii) $T$ is $\mathfrak{H}$-relatively Bernoullian,

(iv) $\mathfrak{F} \perp_{\mathfrak{F} \cap \mathfrak{H}} \mathfrak{H}$,

(v) the factor $\mathfrak{F} \cap \mathfrak{H}$ is class-bijective.

Then $\mathfrak{F}$ is $(\mathfrak{F} \cap \mathfrak{H})$-relatively Bernoullian.

Proof. (A) First of all, let us extend Lemma 4.8 to a bit more general case where $h(\gamma, \mathfrak{H})<\infty$ instead of $h(\gamma)<\infty$. To this end we select an increasing sequence $\mathfrak{F}_{1} \subset \mathfrak{F}_{2} \subset \cdots$ of factors of $\gamma$ such that

$$
\bigcup_{n} \mathfrak{F}_{n}=\mathfrak{F}, \quad \mathfrak{F}_{n} \cap \mathfrak{H}=\mathfrak{F} \cap \mathfrak{H} \text { and } h\left(\gamma, \mathfrak{F}_{n} \mid \mathfrak{F} \cap \mathfrak{H}\right)<\infty
$$

By Theorem 4.9, $\gamma \uparrow\left(\mathfrak{F}_{n} \vee \mathfrak{H}\right)$ is $\mathfrak{H}$-relatively Bernoullian. Moreover,

$$
h\left(\gamma, \mathfrak{F}_{n} \vee \mathfrak{H} \mid \mathfrak{H}\right)=h\left(\gamma, \mathfrak{F}_{n} \mid \mathfrak{H}\right) \leq h\left(\gamma, \mathfrak{F}_{n} \mid \mathfrak{F} \cap \mathfrak{H}\right) \stackrel{(4-2)}{<} \infty
$$

Since $h(\gamma, \mathfrak{H})<\infty$, it follows that $h\left(\gamma, \mathfrak{F}_{n} \vee \mathfrak{H}\right)<\infty$. Hence we deduce from Lemma 4.8 and the second formula in $(4-2)$ that $\mathfrak{F}_{n}$ is $(\mathfrak{F} \cap \mathfrak{H})$-relatively Bernoullian. In view of the first formula in $(4-2), \mathfrak{F}$ is $\mathfrak{F} \cap \mathfrak{H}$-relatively Bernoullian.

(B) Now we state and prove the orbital version of the lemma:

let $\alpha, \beta: \mathcal{R} \rightarrow \operatorname{Aut}(Y, \nu)$ be two cocycles of $\mathcal{R}$ such that the following are satisfied:

(i) the product cocycle $\alpha \times \beta: \mathcal{R} \rightarrow$ Aut $(Y \times Y, \nu \times \nu)$ is ergodic,

(ii) the cocycle $\beta \otimes 1: \mathcal{R}(\alpha) \rightarrow \operatorname{Aut}(Y, \nu)$ given by

$$
\beta \otimes 1\left((x, y),\left(x^{\prime}, y^{\prime}\right)\right):=\beta\left(x, x^{\prime}\right)
$$

is Bernoullian.

Then $\beta$ is Bernoullian.

To prove this, pick a 0 -entropy transformation $\theta$ of $X$ which generates $\mathcal{R}$. It is easy to verify that $\left(\theta_{\alpha}\right)_{\beta \otimes 1}=\theta_{\alpha \times \beta}$. Set $(Z, \kappa):=(X \times Y \times Y, \mu \times \nu \times \nu), \gamma:=\theta_{\alpha \times \beta}$, $\mathfrak{H}:=\mathfrak{B}_{X} \otimes \mathfrak{B}_{Y} \otimes \mathfrak{N}_{Y}, \mathfrak{F}:=\mathfrak{B}_{X} \otimes \mathfrak{N}_{Y} \otimes \mathfrak{B}_{Y}$ and apply (A). Notice that the relative disjointness condition (iii) of Lemma 4.8 corresponds now to the product measure $\nu \times \nu$ on $Y \times Y$.

(C) To deduce the lemma from (B), use the same (standard now) argument as in Proposition 4.5 and Theorem 4.9.

We introduce the concept of the strong Pinsker property for countable group actions exactly like it was done in $[\mathrm{Th} 2]$ for $\mathbb{Z}$-actions with finite entropy. 
Definition 4.11. An ergodic free $G$-action $T$ on $\left(X, \mathfrak{B}_{X}, \mu\right)$ satisfies the strong Pinsker property if there are two factors $\mathfrak{F}_{1}$ and $\mathfrak{F}_{2}$ of $T$ such that the following properties are fulfilled

(i) $\mathfrak{F}_{1} \vee \mathfrak{F}_{2}=\mathfrak{B}_{X}$,

(ii) $\mathfrak{F}_{1} \perp \mathfrak{F}_{2}$,

(iii) $T \uparrow \mathfrak{F}_{1}$ is Bernoullian,

(iv) $h\left(T, \mathfrak{F}_{2}\right)=0$.

Now we can extend the main result from [Th2] to amenable group actions of arbitrary (including infinite) entropy (under some restriction related to the classbijectiveness of factors). The idea of the proof is similar to that from [Th2, Proposition] but we use different tools: Theorem 4.9, Lemma 4.10 and [Da3, Theorem $0.4(\mathrm{i})]$ or $[\mathrm{GTW}$.

Theorem 4.12. Let $T$ be an ergodic free $G$-action satisfying the strong Pinsker property and $\mathfrak{F}$ a factor of $T$ such that the factor $\mathfrak{F} \cap \mathfrak{F}_{2}$ is class bijective. (The factor $\mathfrak{F}_{2}$ is "taken" from Definition 4.11.) Then $T\lceil\mathfrak{F}$ satisfies the strong Pinsker property.

Proof. Since $\Pi(T)=\mathfrak{F}_{2}$, we have $\Pi(T \uparrow \mathfrak{F})=\Pi(T) \cap \mathfrak{F}=\mathfrak{F}_{2} \cap \mathfrak{F}$ and hence $T \uparrow \mathfrak{F}$ is $\left(\mathfrak{F} \cap \mathfrak{F}_{2}\right)$-relatively CPE. On the other hand, $h\left(T, \mathfrak{F}_{2} \mid \mathfrak{F} \cap \mathfrak{F}_{2}\right)=h\left(T, \mathfrak{F}_{2}\right)=0$. It follows from the relative Pinsker theorem for amenable group actions (see [GTW] or [Da3, Thorem $0.4(\mathrm{i})]$ ) that

$$
\mathfrak{F} \perp_{\mathfrak{F} \cap \mathfrak{F}_{2}} \mathfrak{F}_{2} .
$$

Next, the factor $\mathfrak{F} \vee \mathfrak{F}_{2}$ is $\mathfrak{F}_{2}$-relatively Bernoullian by Theorem 4.9 . We deduce from this, (4-3) and Lemma 4.10 that $T\left\lceil\mathfrak{F}\right.$ is $\left(\mathfrak{F} \cap \mathfrak{F}_{2}\right)$-relatively Bernoullian. According to Remark 3.6, there exists a Bernoullian $T$-factor $\mathfrak{F}^{\prime} \subset \mathfrak{F}$ such that $\mathfrak{F}^{\prime} \perp\left(\mathfrak{F} \cap \mathfrak{F}_{2}\right)$ and $\mathfrak{F}^{\prime} \vee\left(\mathfrak{F} \cap \mathfrak{F}_{2}\right)=\mathfrak{F}$.

We complete this section by extending some results from a recent paper [Ho] to amenable group actions. Remark at once that these extensions are partial, i.e. not verbatim (see the comment at the end of the section).

Definition 4.13. Let $S$ and $S^{\prime}$ be two ergodic free actions of $G$ on $\left(Y, \mathfrak{B}_{Y}, \nu\right)$ and $\left(Y^{\prime}, \mathfrak{B}_{Y}^{\prime}, \nu^{\prime}\right)$ respectively and $\pi: Y \rightarrow X$ and $\pi^{\prime}: Y^{\prime} \rightarrow X$ two factor maps intertwining $S$ or $S^{\prime}$ respectively with $T$. The extensions $S \stackrel{\pi}{\rightarrow} T$ and $S^{\prime} \stackrel{\pi^{\prime}}{\rightarrow} T$ of $T$ are called equivalent if there is a measure space isomorphism $\phi:\left(Y, \mathfrak{B}_{Y}, \nu\right) \rightarrow$ $\left(Y^{\prime}, \mathfrak{B}_{Y^{\prime}}, \nu^{\prime}\right)$ such that $\phi \circ \pi^{\prime}=\pi \circ \phi$ and $\phi \circ S_{g}=S_{g}^{\prime} \circ \phi$ for each $g \in G$.

It is well known that every extension $S \stackrel{\pi}{\rightarrow} T$ can be represented in a skew product form. Actually, let $\mathcal{R}$ stand for the $T$-orbit equivalence relation on $X$. Then there exists a cocycle $\alpha: \mathcal{R} \rightarrow \operatorname{Aut}(Y, \nu)$ such that $S \stackrel{\pi}{\rightarrow} T$ is equivalent to $T^{\alpha} \stackrel{\pi_{X}}{\rightarrow} T$, where $\pi_{X}: X \times Y \rightarrow X$ is the projection onto the first coordinate. The cocycle $\alpha$ is determined by $S \stackrel{\pi}{\rightarrow} T$ up to cohomology. Moreover, two extensions are equivalent if and only if the corresponding cocycles of $\mathcal{R}$ are cohomologous. It follows, in particular, that equivalent extensions have the same relative entropy.

The following statement is a relative analogue of the Ornstein-Shields [OrS] collection of uncountably many non-isomorphic $K$-transformations of the same entropy. It was proved in $[\mathrm{Sw}]$ and $[\mathrm{Ho}]$. 
Lemma 4.14. There are two Bernoullian transformations $t$ and $t^{\prime}$ and uncountably many factors $\mathfrak{F}_{\theta}, \theta \in \Theta$, of $t$ such that the following are satisfied:

(i) $t$ is $\mathfrak{F}_{\theta}$-relatively $C P E$,

(ii) $h\left(t, \mathfrak{F}_{\theta}\right)=h\left(t^{\prime}\right)$ and hence there exists an isomorphism (conjugacy) $\phi_{\theta}$ of $t \uparrow \mathfrak{F}_{\theta}$ and $t^{\prime}$ (recall that a factor of a Bernoulli shift is itself a Bernoulli shift),

(iii) the extensions $t \rightarrow \phi_{\theta} \circ\left(t \uparrow \mathfrak{F}_{\theta}\right) \circ \phi_{\theta}^{-1}=t^{\prime}, \theta \in \Theta$, are pairwise nonequivalent.

Using our approach we deduce from this

\section{Proposition 4.15.}

(i) For hyperfinite $\mathcal{R}$, there exist uncountably many pairwise non-cohomologous $C P E$-cocycles $\alpha_{\theta}: \mathcal{R} \rightarrow$ Aut $(Y, \nu), \theta \in \Theta$, with the same entropy.

(ii) Let $T$ be an ergodic free action of $G$. Then there exist uncountably many non-equivalent relatively CPE extensions of $T$ with the same entropy.

In contrast to Proposition 4.15(ii), it follows immediately from Theorem 3.11 that

Corollary 4.16. Every two relatively Bernoullian extensions of $T$ with the same relative entropy are equivalent.

Lemma 4.17 [Ho]. Every Bernoullian shift contains a factor with respect to which it is relatively prime.

From this we deduce

\section{Proposition 4.18.}

(i) There exists an ergodic prime cocycle of $\mathcal{R}$.

(ii) Given an ergodic free action $T$ of $G$, there exists an ergodic extension of $T$ which is relatively prime.

\section{Free factors of Small entropy and “absolute" Sinai theorem}

Our main purpose in this section is to prove that every ergodic free action of a countable amenable group has a free (or, equivalently, class bijective) factor of arbitrarily small entropy. At first we do that for several special classes of amenable groups like Abelian, torsion free or locally finite ones. The proof in each of these cases is in some way elementary since it does not use the machinery developed in [OrW]. Then we settle the general case. However to do that we apply one (only) statement from $[\mathrm{OrW}]$ - Rokhlin lemma for countable amenable group actions. We complete this section with the "absolute" Sinai theorem about Bernoullian factors for amenable actions. It follows from its "relative" counterpart $(\S 4)$ and the main theorem here.

For $t \in(0,1)$, we let $E(t):=t \log t+(1-t) \log (1-t)$.

Proposition 5.1. Let $T$ be an ergodic free action of $G$. If one of the following is fulfilled:

(i) $G$ is torsion free,

(ii) given $g \in G$ of finite order, the T-action of the subgroup $\{h \in G \mid g h=h g\}$ is ergodic,

(iii) $G$ is locally finite, 
then for each $\epsilon>0$, there exists a free factor $\mathfrak{F}$ of $T$ with $h(T, \mathfrak{F})<\epsilon$.

Proof. I. Suppose first that (i) holds. Let $\left(\epsilon_{g}\right)_{g \in G}$ be a family of positive reals such that $\sum_{g \in G} \epsilon_{g}<\epsilon$. For each $g \in G, g \neq 1_{G}$, denote by $\mathfrak{A}_{g}$ the sub- $\sigma$-algebra of $T_{g}$-fixed subsets, i.e.

$$
\mathfrak{A}_{g}:=\left\{A \in \mathfrak{B}_{X} \mid T_{g} A=A\right\} .
$$

Clearly, $\mathfrak{A}_{g}$ is $T_{g}$-invariant. Let $\mu=\int \mu_{x} d\left(\mu\left\lceil\mathfrak{A}_{g}\right)(x)\right.$ stand for the $T_{g}$-ergodic decomposition of $\mu$. Then $\mu_{x} \circ T_{g}=\mu_{x}$ for a.e. $x$. Since $g$ is of infinite order and $T$ is free, $\mu_{x}$ is non-atomic. Take a $t_{g} \in(0,1 / 2)$ with $E\left(t_{g}\right)<\epsilon_{g}$. Then we can choose a subset $A_{g} \subset X$ such that

$$
\begin{aligned}
& \mu_{x}\left(A_{g}\right)=t_{g} \text { for all } x \in X, \text { and } \\
& T_{g} A_{g} \cap A_{g}=\emptyset .
\end{aligned}
$$

Now define a partition $P_{g}$ of $X$ and a factor $\mathfrak{F}$ of $T$ by setting $P_{g}:=\left(A_{g}, X \backslash A_{g}\right)$ and $\mathfrak{F}:=\bigvee_{1_{G} \neq g \in G} \bigvee T_{h} P_{g}$. We have

$$
h(T, \mathfrak{F}) \leq \sum_{1_{g} \neq g \in G} H\left(P_{g}\right)=\sum_{1_{G} \neq g \in G} E\left(t_{g}\right)<\sum_{1_{G} \neq g \in G} \epsilon_{g}<\epsilon .
$$

If $T \nmid \mathfrak{F}$ is not free then there exist $B \in \mathfrak{F}$ and $g \in G \backslash\left\{1_{G}\right\}$ such that $\mu(B)>0$ and $T_{g} B^{\prime}=B^{\prime} \bmod \mu \uparrow \mathfrak{F}$ for every subset $B^{\prime} \subset B$ from $\mathfrak{F}$. Clearly, $B \in \mathfrak{A}_{g}$ and hence $\mu_{x}(B)=0$ or 1 for a.e. $x$. We deduce from this and (5-1) that

$$
\mu\left(B \cap A_{g}\right)=\int \mu_{x}\left(B \cap A_{g}\right) d\left(\mu\left\lceil\mathfrak{A}_{g}\right)(x)=\int_{B} \mu_{x}\left(A_{g}\right) d\left(\mu\left\lceil\mathfrak{A}_{g}\right)(x)=t_{g} \mu(B)>0 .\right.\right.
$$

Moreover, $A_{g} \in P_{g} \subset \mathfrak{F}$ and $T_{g}\left(B \cap A_{g}\right) \cap\left(B \cap A_{g}\right)=\emptyset$ by (5-2), a contradiction.

II. Assume now that (ii) is satisfied. Then we need to modify slightly our argument. Namely, if $g$ is of infinite order, we define $A_{g}$ just like in the previous case. But if $g$ is a torsion then we let $A_{g}$ to be an arbitrary subset of measure $t_{g}$ satisfying (5-2). Define $\mathfrak{F}$ as above. Then $h(T, \mathfrak{F})<\epsilon$ and $T_{g}\lceil\mathfrak{F}$ has no fixed points $\bmod \mu \uparrow \mathfrak{F}$ for each $g$ of infinite order (see (I)). If $g$ is of finite order, then it is easy to deduce from (i) that either $T_{g}\left\lceil\mathfrak{F}\right.$ has no fixed points or $T_{g} \uparrow \mathfrak{F}=I d \bmod \mu\lceil\mathfrak{F}$. The last contradicts to (5-2) since $A_{g} \in \mathfrak{F}$.

III. If (iii) holds then we can assume without loss of generality that $G=$ $\bigcup_{n=1}^{\infty} G_{n}$, where $G_{1} \subset G_{2} \subset \cdots$ is an increasing sequence of finite subgroups with $\sum_{n=1}^{\infty} E\left(1 / \# G_{n}\right)<\epsilon$. Then there exists a sequence $X_{n}$ of subsets in $X$ such that $T_{g} X_{n}, g \in G_{n}$, are disjoint and $\bigcup_{g \in G_{n}} T_{g} X_{n}=X$.

We define a partition $P_{n}$ of $X$ and a factor $\mathfrak{F}$ of $T$ by setting $P_{n}:=\left(X_{n}, X \backslash X_{n}\right)$ and $\mathfrak{F}:=\bigvee_{n=1}^{\infty} \bigvee_{g \in G} T_{g} P_{n}$. It is easy to verify that $\mathfrak{F}$ is as desired.

As it was already said, we are unable to prove Proposition 5.1 in the general setting - i.e. without restrictions (i)-(iii) - in a way avoiding the use of Rokhlin lemma from $[\mathrm{OrW}$. To apply this lemma we need first to recall two concepts from [OrW].

\section{Definition 5.2.}

(i) Let $K$ be a finite subset of $G$ and $\epsilon>0$. A subset $F \subset G$ is $[K, \epsilon]$-invariant if $\#\{f \in F \mid K f \subset F\}>(1-\epsilon) \# F$.

(ii) Subsets $X_{1}, \ldots, X_{n}$ of $X$ are $\epsilon$-disjoint if there are subsets $X_{i}^{\prime} \subset X_{i}$ such that $\mu\left(X_{i}^{\prime}\right)>(1-\epsilon) \mu\left(X_{i}\right)$ and $X_{1}^{\prime}, \ldots, X_{n}^{\prime}$ are (pairwise) disjoint. 
Lemma 5.3. Given $K \subset G$ and $\epsilon>0$ with $E(1 / \# K)<\epsilon / 8$, there exists a finite partition $P$ of $X$ such that the following are satisfied:

(i) $h(T, P)<\epsilon$

(ii) for every $h \in K \backslash\left\{1_{G}\right\}$ and $A \subset X, \mu(A)>6 \epsilon$, there is a subset $C \in$ $\bigvee_{g \in G} T_{g} P$ with $\mu\left(T_{h}(A \cap C) \backslash(A \cap C)\right)>0$.

Proof. It follows from the Rokhlin lemma for amenable group actions [OrW] that there are a sequence of finite $G$-subsets $F_{1} \subset F_{2} \subset \cdots \subset F_{n}$ and a family of measurable $X$-subsets $B_{i}^{j}, j=1, \ldots, J_{i}, i=1, \ldots, n$ such that the following properties are satisfied:

(1) $K \subset F_{1}$,

(2) $F_{i}$ is $\left[K, \epsilon^{2}\right]$-invariant, $i=1, \ldots, n$,

(3) the subsets $T_{g} B_{i}^{j}, g \in F_{i}$, are disjoint,

(4) the subsets $\bigcup_{g \in F_{i}} T_{g} B_{i}^{j}, j=1, \ldots, J_{n}$ are $\epsilon^{2}$-disjoint,

(5) the subsets $\bigcup_{j=1}^{J_{i}} \cup T_{g} B_{i}^{j}, i=1, \ldots, n$ are disjoint,

(6) $\mu\left(\bigcup_{i=1}^{n} \bigcup_{j=1}^{J_{i}} \bigcup_{g \in F_{i}} T_{g} B_{i}^{j}\right)>1-\epsilon$,

(7) the subsets $\bigcup_{g \in F_{i-1}} T_{g} B_{i}^{j}, j=1, \ldots, J_{i}$, are disjoint, where $F_{0}:=K$.

Remark that this statement is stronger than the original Rokhlin lemma from [OrW] which is without (7). Our version can be proved in a similar way as [Ro, Lemma 2] where the following is claimed instead of (7):

the subsets $\bigcup_{g \in K} T_{g} B_{i}^{j}, j \in J_{i}$, are disjoint.

(We assume that $F_{i}$ is much "larger" than $F_{i-1}, i=1, \ldots, n$.)

Define a finite partition $P=\left(P_{0}, \ldots, P_{n}\right)$ of $X$ by setting $P_{i}:=\bigcup_{j=1}^{J_{i}} B_{i}^{j}, j=$ $1, \ldots, n$ and $P_{0}:=X \backslash \bigcup_{i=1}^{n} P_{i}$.

We first estimate the entropy $h(T, P)$. Let $F^{\prime} \subset F$ be two subsets of $G$ such that

$$
\begin{aligned}
& h(T, P) \leq \frac{1}{\# F} H\left(\bigvee_{g \in F} T_{g} P\right)+\epsilon / 2, \\
& F_{n} F^{\prime} \subset F \text { and } \# F^{\prime}>\left(1-\epsilon^{\prime}\right) \# F,
\end{aligned}
$$

where $\epsilon^{\prime}:=\epsilon /(4 \log (n+1))$. Denote by $N$ the number of atoms of the partition $\bigvee_{g \in F} T_{g} P$. Clearly, $N$ is just the number of $(P, F)$-names of the points of $X$. Recall that the $(P, F)$-name of $x \in X$ is a map $\theta_{x}: F \rightarrow\{0, \ldots, n\}$ such that $\theta_{x}(g)=i$ if $T_{g} x \in P_{i}$. We select a sequence $f_{1}, \ldots, f_{n} \in G$ with $f_{1}:=1_{G}$ and $f_{i} \in F_{i-1} \backslash F_{i-2}$, $i=2, \ldots, n$ and set

$$
\begin{aligned}
& S\left(\theta_{x}\right):=\left\{g \in F^{\prime} \mid \theta_{x}(g) \neq 0\right\} \\
& R\left(\theta_{x}\right):=\left\{f_{\theta_{x}(g)} g \mid g \in S\left(\theta_{x}\right)\right\} .
\end{aligned}
$$

It is easy to deduce from (3), (5) and (7) the following assertion.

Claim A. If $g, h \in S\left(\theta_{x}\right)$ and $g \neq h$ then $F_{\theta_{x}(g)-1} g \cap F_{\theta_{x}(h)-1} h=\emptyset$.

It follows immediately that

$$
\# S\left(\theta_{x}\right)=\# R\left(\theta_{x}\right)
$$


Claim B. If $S\left(\theta_{x}\right)=S\left(\theta_{y}\right)$ and $R\left(\theta_{x}\right)=R\left(\theta_{y}\right)$ then $\theta_{x}=\theta_{y}$ on $F^{\prime}$.

Actually, if $\theta_{x}(g)>\theta_{y}(g)$ for some $g \in S\left(\theta_{x}\right)$ then $\left\{f_{\theta_{y}(g)} g, f_{\theta_{x}(g)} g\right\} \in F_{\theta_{x}(g)-1} g$. Since $R\left(\theta_{y}\right)=R\left(\theta_{x}\right)$, there exists $h \in S\left(\theta_{x}\right)$ with $f_{\theta_{y}(g)} g=f_{\theta_{x}(h)} h \in F_{\theta_{x}(h)-1} h$. It follows from Claim A that $h=g$ and hence $\theta_{x}(h)=\theta_{y}(g)$, a contradiction.

Next, from (1), Claim A and (5-4) we deduce that the subsets $K f, f \in S\left(\theta_{x}\right)$, are disjoint and inside $F$. Hence

$$
\max _{x \in X} \# S\left(\theta_{x}\right) \leq \frac{\# F}{\# K} .
$$

It follows from (5-5), (5-6) and Claim B that

$$
\#\left\{\theta_{x}\left|F^{\prime}\right| x \in X\right\} \leq \sum_{i=0}^{\# F / \# K}\left(\begin{array}{c}
\# F \\
i
\end{array}\right)^{2} \leq\left(\sum_{i=0}^{\# F / \# K}\left(\begin{array}{c}
\# F \\
i
\end{array}\right)\right)^{2} \leq 2^{2 \# F \cdot E(1 / \# K)} .
$$

This and (5-4) yield

$$
\begin{aligned}
\#\left\{\theta_{x} \mid x \in X\right\} & \leq \#\left\{\theta_{x} \uparrow F^{\prime} \mid x \in X\right\} \cdot \#\left\{\theta_{x} \uparrow\left(F \backslash F^{\prime}\right) \mid x \in X\right\} \\
& \leq 2^{2 \# F \cdot E(1 / \# K)}(n+1)^{\epsilon^{\prime} \# F} .
\end{aligned}
$$

Since

$$
H\left(\bigvee_{g \in F} T_{g} P\right) \leq \log N=\log \left(\#\left\{\theta_{x} \mid x \in X\right\}\right),
$$

we deduce from (5-3) and (5-7) that

$$
h(T, P) \leq 2 E(1 / \# K)+\epsilon^{\prime} \log (n+1)+\epsilon<\frac{\epsilon}{4}+\frac{\epsilon}{4}+\frac{\epsilon}{2}=\epsilon,
$$

as desired.

Now let us prove the second claim of the lemma. Fix $h \in K \backslash\left\{1_{G}\right\}$ and $A \subset X$ with $\mu(A)>6 \epsilon$. It follows from (5) and (6) that there exists $i \in\{1, \ldots, n\}$ such that

$$
\mu\left(A \cap \bigcup_{j=1}^{J_{i}} \bigcup_{g \in F_{i}} T_{g} B_{i}^{j}\right)>5 \epsilon \mu\left(\bigcup_{j=1}^{J_{i}} \bigcup_{g \in F_{i}} T_{g} B_{i}^{j}\right) .
$$

From (4) and (3) we deduce that there are disjoint subsets $D^{j} \subset \bigcup_{g \in F_{i}} T_{g} B_{i}^{j}$, $j=1, \ldots, J_{i}$, with

$$
\mu\left(D^{j}\right)>\left(1-\epsilon^{2}\right) \mu\left(\bigcup_{g \in F_{i}} T_{g} B_{i}^{j}\right)=\left(1-\epsilon^{2}\right) \# F_{i} \mu\left(B_{i}^{j}\right)
$$

Hence there exists a $j$ such that

$$
\mu\left(A \cap \bigcup_{g \in F_{i}} T_{g} B_{i}^{j}\right)>4 \epsilon \# F_{i} \mu\left(B_{i}^{j}\right) .
$$

It follows from (2), (3) and (5-8) that we can find a subset $E \subset D^{j}$ with

$$
\begin{aligned}
& T_{h} E \subset D^{j} \text { and } \\
& \mu(E)>\left(1-3 \epsilon^{2}\right) \# F_{i} \mu\left(B_{i}^{j}\right) .
\end{aligned}
$$


Clearly, the subsets $L_{g}:=E \cap T_{g} B_{i}^{j}, g \in F_{i}$, are disjoint and $\bigcup_{g \in F_{i}} L_{g}=E$.

Let us call an element $g \in F_{i}$ good if $\mu\left(L_{g}\right)>(1-2 \epsilon) \mu\left(B_{i}^{j}\right)$. It is a standard argument to deduce from (5-11) that the number of good elements in $F_{i}$ is more then $(1-2 \epsilon) \# F_{i}$. Hence

$$
\mu\left(\bigcup_{g \text { is good }} L_{g}\right)>(1-2 \epsilon)^{2} \# F_{i} \mu\left(B_{i}^{j}\right)>(1-4 \epsilon) \# F_{i} \mu\left(B_{i}^{j}\right) .
$$

It follows from this and (5-9) that there is a good $g \in F_{i}$ with $\mu\left(A \cap L_{g}\right)>0$. Now we set $C:=\bigcup_{l \in J_{i}} T_{g} B_{i}^{l}$. Clearly, $L_{g} \subset C \in \bigvee_{g \in F_{n}} T_{g} P$. In view of (5-10),

$$
T_{h}\left(A \cap L_{g}\right) \subset T_{h}(A \cap C) \backslash(A \cap C) .
$$

Theorem 5.4. Let $T$ be an ergodic free action of a countable amenable group $G$. Then for each $\epsilon>0$, there exists a free factor $\mathfrak{F}$ of $T$ with $h(T, \mathfrak{F})<\epsilon$.

Proof. Let $K_{1} \subset K_{2} \subset \cdots \subset G$ and $\epsilon_{1}>\epsilon_{2}>\ldots$ be such that $\bigcup_{i} K_{i}=G$, $\sum_{i} \epsilon_{i}<\epsilon$ and $E\left(1 / \# K_{i}\right)<\epsilon_{i} / 8$. We apply Lemma 5.3 to every pair $\left(K_{i}, \epsilon_{i}\right)$ to obtain a sequence $P^{1}, P^{2}, \ldots$ of $X$-partitions satisfying (i) and (ii) of this lemma. Let $\mathfrak{F}:=\bigvee_{i=1}^{\infty} \bigvee_{g \in G}^{\infty} T_{g} P^{i}$. Clearly,

$$
h(T, \mathfrak{F}) \leq \sum_{i=1}^{\infty} H\left(T, P^{i}\right)<\sum_{i=1}^{\infty} \epsilon_{i}<\epsilon .
$$

Suppose that $T\left\lceil\mathfrak{F}\right.$ is not free. Then there exist $h \in G \backslash\left\{1_{G}\right\}$ and $A \in \mathfrak{F}$ such that $\mu(A)>0$ and for each $B \subset A, B \in \mathfrak{F}$, we have $T_{h} B=B \bmod \mu \uparrow \mathfrak{F}$. Choose $i$ so that $h \in K_{i}$ and $\mu(A)>6 \epsilon_{i}$. By Lemma 5.3, there is a subset $C \in \bigvee_{g \in G} T_{g} P^{i} \subset \mathfrak{F}$ with $\mu\left(T_{h}(A \cap C) \backslash(A \cap C)\right)>0$, a contradiction.

Remark that we proved Theorem 5.4 independently of the particular cases of it considered in Proposition 5.1.

Combining the above theorem with Corollary 4.6 we obtain the following version of the absolute Sinai theorem on Bernoullian factors of amenable group actions:

Theorem 5.5. Let $T$ be an ergodic G-action. Then for each finite or countable distribution $I$ with $H(I) \in(0, h(T))$ there exists a Bernoullian factor $\mathfrak{F}$ of $T$ with a Bernoullian generator $Q$ such that $\operatorname{dist} Q=I$. Moreover, there is a Bernoullian $T$-factor $\mathfrak{H}$ of the full entropy, i.e. $h(T \mid \mathfrak{H})=0$. (Notice that in case $h(T)<\infty$ the last formula is equivalent to $h(T, \mathfrak{H})=h(T)$.)

Proof. By Theorem 5.4, there exists a class-bijective factor $\mathfrak{E}$ of $T$ with $h(T, \mathfrak{E})<$ $h(T)-H(I)$. We have $h(T \mid \mathfrak{E})=h(T)-h(T, \mathfrak{E})>H(I)$. To conclude the proof of the first claim, it remains to apply Corollary 4.6 .

Now let us prove the second assertion of the proposition. By Theorem 5.4, we can find a class-bijective factor $\mathfrak{E}$ of $T$ with $h(T, \mathfrak{E})<h(T) / 2$. It follows from Corollary 4.6 that there is a Bernoullian factor $\mathfrak{F}^{\prime}$ of $T$ with $h\left(T, \mathfrak{F}^{\prime}\right)<h(T) / 2$. By the entropy addition formula, $h\left(T \mid \mathfrak{F}^{\prime}\right) \geq h(T) / 2$. Since a Bernoullian factor is class-bijective we deduce from Corollary 4.6 that there exists a Bernoullian factor $\mathfrak{F}^{\prime \prime}$ such that $\mathfrak{F}^{\prime \prime} \perp \mathfrak{F}^{\prime}$ and $h\left(T \mid \mathfrak{F} \vee \mathfrak{F}^{\prime \prime}\right)=0$. Clearly, $\mathfrak{F}^{\prime} \vee \mathfrak{F}^{\prime \prime}$ is a Bernoullian factor of $T$ and we are done. 


\section{Bernoullian factors that SPAN the $\sigma$-Algebra}

Our purpose in this section is to prove

Theorem 6.1. Let $T$ be an ergodic action of a countable amenable group $G$ on $\left(X, \mathfrak{B}_{X}, \mu\right)$ with $0<h(T) \leq \infty$. Then for every $\epsilon>0$, there are three Bernoullian factors $\mathfrak{F}_{0}, \mathfrak{F}_{1}$ and $\mathfrak{F}_{2}$ of $T$ such that $\mathfrak{F}_{0} \vee \mathfrak{F}_{1} \vee \mathfrak{F}_{2}=\mathfrak{B}_{X}$ and $h\left(T \backslash \mathfrak{F}_{i}\right)<\epsilon, i=1,2$.

This extends the main result of $[\mathrm{STh}]$ proved there for $\mathbb{Z}$-actions with finite entropy to arbitrary amenable group actions.

We reproduce here Lemma 1 (with the proof) from [STh] in a slightly modified form.

Lemma 6.2. Let $\gamma$ be an ergodic transformation of $\left(X, \mathfrak{B}_{X}, \mu\right)$ and $\mathfrak{B}_{1}, \mathfrak{B}_{2}$ two factors of $\gamma$ such that

(i) $\mathfrak{B}_{1}$ is Bernoullian and $h\left(\gamma, \mathfrak{B}_{1}\right)=1$,

(ii) $h\left(\gamma, \mathfrak{B}_{2}\right)<1$,

(iii) $\mathfrak{B}_{1} \perp \mathfrak{B}_{2}$.

Then there exists a Bernoullian $\gamma$-factor $\mathfrak{B}_{3}$ such that $\mathfrak{B}_{3} \perp \mathfrak{B}_{2}, \mathfrak{B}_{3} \vee \mathfrak{B}_{1} \supset \mathfrak{B}_{2}$ and $h\left(\gamma, \mathfrak{B}_{3}\right) \leq 1$.

Proof. It follows from (ii) and the Krieger theorem $[\mathrm{Kr}]$ that $\gamma \uparrow \mathfrak{B}_{2}$ has a 2element generating partition $\left(A_{1}, A_{2}\right)$. By the Ornstein theorem [Or1], there exists an independent generator $\left(B_{1}, B_{2}\right)$ for $\gamma \uparrow \mathfrak{B}_{1}$ such that $\mu\left(B_{1}\right)=\mu\left(B_{2}\right)=0.5$. We define a 2-element partition $\left(C_{1}, C_{2}\right)$ of $X$ by setting $C_{1}:=\left(A_{1} \cap B_{1}\right) \cup\left(A_{2} \cap B_{2}\right)$. Clearly, $\left(C_{1}, C_{2}\right) \vee\left(B_{1}, B_{2}\right) \supset\left(A_{1}, A_{2}\right)$. Let $\mathfrak{B}_{3}$ stand for the $\gamma$-factor generated by $\left(C_{1}, C_{2}\right)$. It is easy to verify that $\mathfrak{B}_{3}$ is as desired.

Lemma 6.3. Let $\alpha$ be an ergodic cocycle of $\mathcal{R}$ with $h(\alpha)>2$. Let $\mathfrak{A}$ be a factor of $\alpha$ with $h(\alpha, \mathfrak{A})<1$. Then there are two Bernoullian factors $\mathfrak{F}_{1}$ and $\mathfrak{F}_{2}$ of $\alpha$ such that $\mathfrak{F}_{1} \vee \mathfrak{F}_{2} \supset \mathfrak{A}$ and $h\left(\alpha, \mathfrak{F}_{i}\right) \leq 1, i=1,2$.

Proof. Let $\gamma$ be a zero entropy transformation of $X$ generating $\mathcal{R}$. Then

$$
\begin{aligned}
h\left(\gamma_{\alpha} \mid \mathfrak{A}\right) & =h\left(\gamma_{\alpha}\right)-h\left(\gamma_{\alpha}, \mathfrak{A}\right) \\
& =h\left(\gamma_{\alpha} \mid \mathfrak{B}_{X} \otimes \mathfrak{N}_{Y}\right)-h\left(\gamma_{\alpha}, \mathfrak{A} \mid \mathfrak{B}_{X} \otimes \mathfrak{N}_{Y}\right) \\
& =h(\alpha)-h(\alpha, \mathfrak{A})>1
\end{aligned}
$$

By Corollary 4.6, there exists a Bernoullian factor $\mathfrak{B}_{1}$ of $\gamma_{\alpha}$ with $h\left(\gamma_{\alpha}, \mathfrak{B}_{1}\right)=1$ and $\mathfrak{B}_{1} \perp \mathfrak{A}$. Moreover, $h\left(\gamma_{\alpha}, \mathfrak{A}\right)=h(\alpha, \mathfrak{A})<1$. Then it follows from Lemma 6.2 that there is a Bernoullian $\gamma_{\alpha}$-factor $\mathfrak{B}_{2}$ such that

$$
\mathfrak{B}_{2} \perp \mathfrak{A}, \mathfrak{B}_{1} \vee \mathfrak{B}_{2} \supset \mathfrak{A} \text { and } h\left(\gamma_{\alpha}, \mathfrak{B}_{2}\right) \leq 1 .
$$

Clearly, each of $\mathfrak{B}_{1}$ and $\mathfrak{B}_{2}$ is independent of $\mathfrak{B}_{X} \otimes \mathfrak{N}_{Y}$ since $\mathfrak{A} \supset \mathfrak{B}_{X} \otimes \mathfrak{N}_{Y}$. Now we set $\mathfrak{F}_{i}:=\mathfrak{B}_{i} \vee\left(\mathfrak{B}_{X} \otimes \mathfrak{N}_{Y}\right), i=1,2$. Then $\mathfrak{F}_{1}$ and $\mathfrak{F}_{2}$ are $\left(\mathfrak{B}_{X} \otimes \mathfrak{N}_{Y}\right)$-relatively Bernoullian factors of $\gamma_{\alpha}$ and hence Bernoullian factors of $\alpha$ (see Proposition 3.8). Moreover, $h\left(\alpha, \mathfrak{F}_{i}\right) \leq h\left(\gamma_{\alpha}, \mathfrak{B}_{i}\right) \leq 1$, as desired.

The following statement is crucial in the proof of Theorem 6.1. We show here that the original idea of Thouvenot and Smorodinsky to make use of the induced transformation (which fails for actions of the groups other than $\mathbb{Z}$ ) can be successfully replaced by its orbital counterpart - just consider the induced equivalence relation and restrict the cocycle to it. 
Lemma 6.4. Let $Q=\left(A, A_{1}, \ldots, A_{k}\right)$ be a finite partition of $X$ and $\mathfrak{P}$ a sub- $\sigma$ algebra of $\left(\mathfrak{B}_{X} \otimes \mathfrak{B}_{Y}\right) \uparrow(A \times Y)$. Let $\mathfrak{P}^{\prime}$ stand for the "associated" sub- $\sigma$-algebra of $\mathfrak{B}_{X} \otimes \mathfrak{B}_{Y}$, i.e. the smallest sub- $\sigma$-algebra containing $Q \otimes \mathfrak{N}_{Y}$ and such that $\mathfrak{P}^{\prime} \uparrow(A \times Y)=\mathfrak{P}$. Denote by $\alpha^{A}$ the restriction of $\alpha$ to the induced equivalence relation $\mathcal{R} \cap(A \times A)$ on the standard probability space $\left(A, \mathfrak{B}_{X}\left\lceil A, \frac{\mu}{\mu(A)}\right)\right.$. Then

(i) $\mathfrak{P}$ is a generator for $\alpha^{A}$ if and only if $\mathfrak{P}^{\prime}$ is a generator for $\alpha$.

(ii) $h\left(\alpha^{A}, \mathfrak{P}\right)=h\left(\alpha, \mathfrak{P}^{\prime}\right) / \mu(A)$.

(iii) $h\left(\alpha^{A}\right)=h(\alpha) / \mu(A)$.

(iv) If $h(\alpha)<\mu(A)$ then there is a two-element partition of $A \times Y$ such that the "associated" partition of $X \times Y$ is a generator for $\alpha$ (as usual, we identify a finite partition with the sub- $\sigma$-algebra that it generates).

(v) Let $T$ be a Bernoullian $G$-action on $X$ generating $\mathcal{R}$ and $Q$ an independent generator of $T$. Then $\mathfrak{P}$ is Bernoullian for $\alpha^{A}$ if and only if $\mathfrak{P}^{\prime}$ is independent under $T^{\alpha}$.

Proof. (i) Let $\mathfrak{P}$ be a generator for $\alpha^{A}$. Then

$$
\bigvee_{x^{\prime} \in \mathcal{R}(x) \cap A} \alpha\left(x, x^{\prime}\right) \mathfrak{P}_{x^{\prime}}=\mathfrak{B}_{Y} \text { for a. a. } x \in A
$$

Notice that

$$
\mathfrak{P}_{x^{\prime}}^{\prime}= \begin{cases}\mathfrak{P}_{x^{\prime}} & \text { for } x^{\prime} \in A \\ \mathfrak{N}_{Y} & \text { otherwise. }\end{cases}
$$

Since $\mathcal{R}$ is ergodic, for a.e. $x \in X$ there exists $z \in \mathcal{R}(x) \cap A$. Hence

$$
\bigvee_{x^{\prime} \in \mathcal{R}(x)} \alpha\left(x, x^{\prime}\right) \mathfrak{P}_{x^{\prime}}^{\prime} \supset \alpha(x, z) \bigvee_{x^{\prime} \in \mathcal{R}(z) \cap A} \alpha\left(z, x^{\prime}\right) \mathfrak{P}_{x^{\prime}}=\mathfrak{B}_{Y}
$$

This means that $\mathfrak{P}^{\prime}$ is a generator for $\alpha$. The converse claim follows immediately from $(6-1)$.

(ii) Take a finite $\mathfrak{P}$-measurable partition $P$ of $A \times Y$ and denote by $P^{\prime}$ the "associated" finite partition of $X \times Y$. Clearly that if $\left(\mathcal{R}_{n}\right)_{n \geq 1}$ is a filtration of $\mathcal{R}$ then $\left(\mathcal{R}_{n} \cap(A \times A)\right)_{n \geq 1}$ is a filtration for $\mathcal{R} \cap(A \times A)$. By Proposition 1.2,

$$
h\left(\alpha, P^{\prime}\right)=\lim _{n \rightarrow \infty} \frac{1}{\# \mathcal{R}_{n}(x)} H\left(\bigvee_{x^{\prime} \in \mathcal{R}_{n}(x)} \alpha\left(x, x^{\prime}\right) P_{x^{\prime}}^{\prime}\right) \text {, for a.e } x \in A .
$$

Since $\mathcal{R}$ is ergodic,

$$
\frac{\#\left(\mathcal{R}_{n}(x) \cap A\right)}{\# \mathcal{R}_{n}(x)} \rightarrow \mu(A) \text { as } n \rightarrow \infty .
$$

From this, (6-1) and (6-2) we deduce that

$$
h\left(\alpha, P^{\prime}\right)=\lim _{n \rightarrow \infty} \frac{\mu(A)}{\#\left(\mathcal{R}_{n}(x) \cap A\right)} H\left(\bigvee_{x^{\prime} \in \mathcal{R}_{n}(x) \cap A} \alpha^{A}\left(x, x^{\prime}\right) P_{x^{\prime}}\right)=\mu(A) h\left(\alpha^{A}, P\right) .
$$


Consider now an increasing sequence of finite $(A \times Y)$-partitions $P_{1} \subset P_{2} \subset \cdots$ with $\bigvee_{n=1}^{\infty} P_{n}=\mathfrak{P}$ and pass to the limit in $h\left(\alpha, P_{n}^{\prime}\right)=\mu(A) h\left(\alpha^{A}, P_{n}\right)$.

(iii) follows from (i), (ii) and Lemma 2.4.

(iv) It follows from (ii) that $h\left(\alpha^{A}\right)<1$. By Lemma 2.6, there exists a 2-element generator $\left(P_{1}, P_{2}\right)$ for $\alpha$. It remains to apply (i).

(v) Let $P$ and $P^{\prime}$ be like in the proof of (ii). Since $P^{\prime} \supset Q \otimes \mathfrak{N}_{Y}$, we deduce from the Pinsker formula for amenable group actions, Lemma 1.3, and (ii) that

$$
\begin{aligned}
h\left(T^{\alpha}, P^{\prime}\right) & =h\left(T^{\alpha}, Q \otimes \mathfrak{N}_{Y}\right)+h\left(T^{\alpha}, P^{\prime} \mid \mathfrak{B}_{X} \otimes \mathfrak{N}_{Y}\right) \\
& =h(T, Q)+h\left(\alpha, P^{\prime}\right) \\
& =H(Q)+\mu(A) h\left(\alpha^{A}, P\right) .
\end{aligned}
$$

On the other hand, $H\left(P^{\prime}\right)=H(Q)+\mu(A) H(P)$. Hence $h\left(T^{\alpha}, P^{\prime}\right)=H\left(P^{\prime}\right)$ if and only if $h\left(\alpha^{A}, P\right)=H(P)$. Thus, $P^{\prime}$ is independent under $T^{\alpha}$ if and only if $P$ is Bernoullian for $\alpha^{A}$. Hence $\mathfrak{P}^{\prime}$ is independent under $T^{\alpha}$ if and only if $\mathfrak{P}$ is Bernoullian for $\alpha^{A}$.

Notice in advance that to demonstrate Theorem 6.1 we need only a part of the material from Lemma 6.4, namely: (i)-(iii), the "only if" part of (v) and the case $h(\alpha)=0$ from (iv), and all of that under the condition $k=1$ and $\mathfrak{P}$ is finite. The "surplus" of the lemma will be used in the next section.

Proof of Theorem 6.1. Let $I=\left(I_{1}, I_{2}\right)$ be a distribution such that the following properties are satisfied:

(a) $I_{1}<h(T) / 4$,

(b) $H(I)<h(T) / 2$, and

(c) the entropy of the distribution $I^{\prime}:=\left(I_{1} / 2, I_{1} / 2, I_{2}\right)$ is less than $\epsilon$.

By Theorem 5.5, there exists an independent partition $\left(P_{0}, P_{1}\right)$ of $X$ with $\mu\left(P_{i}\right)=$ $I_{i}, i=0,1$. Denote by $\mathfrak{F}$ the Bernoullian factor of $T$ generated by this partition. Then

$$
h(T \mid \mathfrak{F})=h(T)-H(I) \stackrel{(\mathrm{b})}{>} h(T) / 2 \stackrel{(\mathrm{a})}{>} 2 \mu\left(P_{0}\right) .
$$

It follows from Corollary 4.6 that there exists a Bernoullian factor $\mathfrak{F}_{0}$ of $T$ with $h\left(T \mid \mathfrak{F}_{0}\right)=0$ and $\mathfrak{F}_{0} \supset \mathfrak{F}$.

Consider first the extension $T \rightarrow T \uparrow \mathfrak{F}_{0}$ and the corresponding (extending) cocycle of the $\left(T \uparrow \mathfrak{F}_{0}\right)$-orbit equivalence relation - see the remark just below Definition 5.2. From Lemma 1.3 we deduce that the entropy of this cocycle is 0 . Hence by Lemma 6.4(iv) and Remark 2.3, the subset $P_{0}$ can be partitioned into two subsets $P_{2}$ and $P_{3}$ in such a way that

$$
\mathfrak{F}_{0} \vee \bigvee_{g \in G} T_{g}\left(P_{1}, P_{2}, P_{3}\right)=\mathfrak{B}_{X}
$$

Now let $\mathcal{R}$ stand for the $(T \uparrow \mathfrak{F})$-orbit equivalence relation on $(X, \mathfrak{F}, \mu \uparrow \mathfrak{F})$ and $\alpha$ for the cocycle of $\mathcal{R}$ corresponding to the extension $T \rightarrow T \uparrow \mathfrak{F}$. By Lemma 1.3 and (6-3), we have

$$
\begin{gathered}
h(\alpha)=h(T \mid \mathfrak{F})>2 \mu\left(P_{0}\right) . \\
26
\end{gathered}
$$


According to Lemma 6.4(iii), this implies $h\left(\alpha^{P_{0}}\right)>2$. Let $\mathfrak{A}$ stand for the factor of $\alpha^{P_{0}}$ generated by the partition $\left(P_{2}, P_{3}\right)$ (see Remark 4.3(iv)). Clearly, $h(\alpha, \mathfrak{A}) \leq$ $\log 2=1$. Hence we can deduce from Lemma 6.3 that there are two Bernoullian factors $\mathfrak{E}_{1}$ and $\mathfrak{E}_{2}$ of $\alpha^{P_{0}}$ with

$$
\mathfrak{E}_{1} \vee \mathfrak{E}_{2} \supset \mathfrak{A}
$$

and $h\left(\alpha^{P_{0}}, \mathfrak{E}_{i}\right) \leq 1=\log 2, i=1,2$. By Lemma 3.14, there exist Bernoullian 2-element generators $Q^{1}$ and $Q^{2}$ for $\mathfrak{E}_{1}$ and $\mathfrak{E}_{2}$ respectively. It follows from Lemma 6.4(v) that the partitions $\left(Q^{1}, P_{1}\right)$ and $\left(Q^{2}, P_{1}\right)$ are independent under $T$. Moreover, the entropy of each of these partitions does not exceed $h\left(I^{\prime}\right)$ which is less than $\epsilon$ according to (c). Next, we deduce from (6-5) that

$$
\bigvee_{g \in G} T_{g}\left(Q^{1}, P_{1}\right) \vee \bigvee_{g \in G} T_{g}\left(Q^{2}, P_{1}\right) \supset \mathfrak{A} \supset\left\{P_{2}, P_{3}\right\}
$$

This and (6-4) yield

$$
\bigvee_{g \in G} T_{g}\left(Q^{1}, P_{1}\right) \vee \bigvee_{g \in G} T_{g}\left(Q^{2}, P_{1}\right) \vee \mathfrak{F}_{0}=\mathfrak{B}_{X}
$$

as desired.

\section{ORNSTEIN-WEISS ISOMORPHISM THEOREM AND ABSOLUTE GENERATORS FOR AMENABLE ACTIONS}

In this section we provide a new short (orbital) proof of the main result from $[\mathrm{OrW}]$ for countable amenable groups, namely the isomorphism theorem for Bernoullian actions of such groups with the same entropy (under a certain restriction in the finite entropy case). To do that we use the "inducing trick" for cocycles of equivalence relations elaborated in $\S 6$. Observe however that the results of $\S 5$ (and hence the Rokhlin lemma for amenable group actions from [OrW]) are applied nowhere in our proof. Thus our proof of the isomorphism theorem is completely independent of that from [OrW]. Notice also that we settle the both cases of the Ornstein-Weiss theorem (of finite and infinite entropy) at one blow.

We complete the section with another application of the "cocycle inducing" technique - a new short comparatively with [Ro] (orbital) proof of the "absolute" Krieger theorem about finite generators for amenable actions is demonstrated. Remark that we improve the estimation of the cardinality of the generators obtained previously in [Ro]. The "absolute" Rokhlin theorem about countable generators for amenable actions of infinite entropy-i.e. an infinite counterpart of the Krieger theorem - is also included here.

Theorem 7.1. Let $T$ and $T^{\prime}$ be two Bernoullian G-actions. They are conjugate if one of the following is satisfied:

(i) $h(T)=h\left(T^{\prime}\right)=\infty$,

(ii) $h(T)=h\left(T^{\prime}\right)<\infty$ and each of $T$ and $T^{\prime}$ has an independent generator with at least 3 elements (as a partition). 
Proof. We first prove that for any sufficiently small $\epsilon>0$, there exists a countable partition $P=\left(P_{1}, P_{2}, \ldots\right)$ of $X$ which is an independent generator for $T$ and $\mu\left(P_{1}\right)=\epsilon$.

Actually, let a sub- $\sigma$-algebra $\mathfrak{Q}^{\prime} \subset \mathfrak{B}_{X}$ be an independent generator of $T$. Without loss of generality we may assume that $\mathfrak{Q}^{\prime}$ is countable (as a partition). Actually, if it is not the case we can represent $\mathfrak{Q}^{\prime}$ as $\mathfrak{Q}_{1}^{\prime} \vee \mathfrak{Q}_{2}^{\prime}$ in such a way that $\mathfrak{Q}_{1}^{\prime} \perp \mathfrak{Q}_{2}^{\prime}$ and $\mathfrak{Q}_{1}^{\prime}$ is countable. Hence $T$ is isomorphic to the Cartesian product of two Bernoullian factors $\mathfrak{B}_{1}$ and $\mathfrak{B}_{2}$ generated by $\mathfrak{Q}_{1}$ and $\mathfrak{Q}_{2}$ respectively. It follows that $T$ is $\mathfrak{B}_{1}$-relatively Bernoullian. hence by Proposition 3.13 there exists a countable $\mathfrak{B}_{1}$-relatively Bernoullian generator $\mathfrak{Q}_{3}^{\prime}$ for $T$. Clearly, $\mathfrak{Q}_{1}^{\prime} \vee \mathfrak{Q}_{3}^{\prime}$ is a countable independent generator for $T$.

It follows from (i) or (ii) that there exists a two set partition $(Q, X \backslash Q)$ of $X$ and a sub- $\sigma$-algebra $\mathfrak{Q}$ of $\mathfrak{B}_{X} \uparrow Q$ such that $\mathfrak{Q}^{\prime}$ is the smallest sub- $\sigma$-algebra containing $(Q, X \backslash Q)$ and whose restriction to $Q$ is $\mathfrak{Q}$. Denote by $\mathfrak{F}$ the $T$-factor generated by the (independent) partition $(Q, X \backslash Q)$. Let $\alpha$ stand for the cocycle corresponding to the extension $T \rightarrow T \uparrow \mathfrak{F}$. (It is defined on the $(T \uparrow \mathfrak{F}$ )-orbit equivalence relation.) By Lemma 6.4 (i,v), the induced cocycle $\alpha^{Q}$ is Bernoullian and $h\left(\alpha^{Q}\right)>0$. Then it is easy to deduce from Lemma 3.14 that for every $\epsilon>0$ such that $E(\epsilon)<h\left(\alpha^{Q}\right)$ there exists a countable Bernoullian generator $\left(P_{1}, P_{2}, \ldots\right)$ for $\alpha^{Q}$ with $\mu\left(P_{1}\right)=\epsilon$. Apply again Lemma 6.4(i,v) to conclude that the countable $X$-partition $P:=\left(X \backslash Q_{1}, P_{1}, P_{2}, \ldots\right)$ is an independent generator for $T$, as desired.

Now let countable partitions $P=\left(P_{1}, P_{2}, \ldots\right)$ and $P^{\prime}=\left(P_{1}^{\prime}, P_{2}^{\prime}, \ldots\right)$ of $X$ be independent generators for $T$ and $T^{\prime}$ respectively. By the above claim, we can assume without loss of generality that $\mu\left(P_{1}\right)=\mu^{\prime}\left(P_{1}^{\prime}\right)$. Denote by $\alpha$ and $\alpha^{\prime}$ the cocycles corresponding to the extensions $T \rightarrow T \uparrow\left(\bigvee_{g \in G} T_{g}\left(P_{1}, X \backslash P_{1}\right)\right)$ and $T^{\prime} \rightarrow$ $T^{\prime} \uparrow\left(\bigvee_{g \in G} T_{g}^{\prime}\left(P_{1}^{\prime}, X^{\prime} \backslash P_{1}^{\prime}\right)\right.$ respectively. By Lemma $6.4(\mathrm{v})$, the induced cocycles $\alpha^{X \backslash P_{1}}$ and $\left(\alpha^{\prime}\right)^{X^{\prime} \backslash P_{1}^{\prime}}$ are Bernoullian. Since $H(P)=h(\alpha)=h\left(\alpha^{\prime}\right)=H\left(P^{\prime}\right)$, it follows that

$$
H\left(P \uparrow\left(X \backslash P_{1}\right)\right)=H\left(P^{\prime} \uparrow\left(X^{\prime} \backslash P_{1}^{\prime}\right)\right)
$$

and hence $h\left(\alpha^{X \backslash P_{1}}\right)=h\left(\left(\alpha^{\prime}\right)^{X^{\prime} \backslash P_{1}^{\prime}}\right)$. By Lemma 3.14, there exist countable Bernoullian generators $Q$ and $Q^{\prime}$ for $\alpha^{X \backslash P_{1}}$ and $\left(\alpha^{\prime}\right)^{X^{\prime} \backslash P_{1}^{\prime}}$ respectively with $\operatorname{dist} Q=$ dist $Q^{\prime}$. But then we can deduce from Lemma 6.4(i,v) that the countable $X$ partitions $\left(P_{1}, Q\right)$ and $\left(P_{1}^{\prime}, Q^{\prime}\right)$ are independent generators for $T$ and $T^{\prime}$ respectively. Since the distributions of these partitions are the same, we are done.

Remark that in the case when each of $T$ and $T^{\prime}$ has a 2-element Bernoullian generator and their entropy are equal then these generators have the same distributions (up to the ordering) and hence the actions are also conjugate.

Theorem 7.2. Let $T$ be an ergodic free $G$-action. If $0<h(T)<\log k$ for some integer $k$ then there exists a finite generator $P$ of $T$ with

$$
\# P= \begin{cases}k, & \text { if } k \text { is not prime } \\ k+1, & \text { otherwise }\end{cases}
$$

If $h(T)=\infty$ then there exists a countable generating partition for $T$.

Proof. (I) Start with the first claim of the theorem. Without loss of generality we may assume that

$$
\begin{gathered}
\log (k-1) \leq h(T)<\log k . \\
28
\end{gathered}
$$


Consider separately 3 cases.

$\left(\mathrm{I}_{1}\right)$ Suppose first that $k=k_{1} k_{2}$ with $k_{1}, k_{2}>1$. By Theorem 5.5 , there exists a Bernoullian factor $\mathfrak{F}$ having a $k_{1}$-element equidistributed Bernoullian generator $P$. Hence

$$
h(T, \mathfrak{F})=\log k_{1} \quad \text { and } \quad h(T \mid \mathfrak{F})=h(T)-h(T, \mathfrak{F})<\log k_{2} .
$$

Since $\mathfrak{F}$ is class-bijective, it follows from Theorem 2.7(ii) that there is an $\mathfrak{F}$-relative generator $Q$ of $T$ with $\# Q=k_{2}$. Clearly, $P \vee Q$ is an (absolute) generator of $T$ and

$$
\#(P \vee Q) \leq \# P \# Q=k_{1} k_{2}=k
$$

as desired.

$\left(\mathrm{I}_{2}\right)$ If $k$ is prime and $k>2$ then we can write $k+1=2 \cdot \frac{k+1}{2}$ and repeat the above argument to obtain the desired estimation.

$\left(\mathrm{I}_{3}\right)$ Suppose now that $k=2$. It is easy to deduce from Theorem 5.5 that there exist a Bernoullian factor $\mathfrak{F}$ and an independent 2-element generator $\left(P_{1}, P_{2}\right)$ of $T \nmid \mathfrak{F}$ such that $h(T \mid \mathfrak{F})<\mu\left(P_{1}\right)$. Let $\alpha$ stand for the cocycle corresponding to the extension $T \rightarrow T\left\lceil\mathfrak{F}\right.$. Then $h(\alpha)<\mu\left(P_{1}\right)$ by Lemma 1.3. Applying Lemma 6.4(iv) like in the proof of Theorem 6.1 we can partition $P_{1}$ into two subsets $A$ and $B$ such that the partition $\left(A, B, P_{2}\right)$ is a generator for $\alpha$ and hence an $\mathfrak{F}$-relative generator for $T$. But this partition (as a $\sigma$-algebra) contains $\left(P_{1}, P_{2}\right)$ which is a generator for $T \nmid \mathfrak{F}$. Hence $\left(A, B, P_{2}\right)$ is an (absolute) generator for $T$.

(II) The second claim of the theorem can be settled in a similar way as $\left(\mathrm{I}_{1}\right)$ with reference to Theorem 2.7(iii) instead of Theorem 2.7(ii).

\section{REFERENCES}

[AR] L. M. Abramov and V. A. Rokhlin, The entropy of a skew product of measure preserving transformations, Vestnik Leningrad. Univ. 7 (1962), 5-13; Amer. Math. Soc. Transl.(2) 48 (1965), 255-265.

[CFW] A. Connes, J. Feldman, and B. Weiss, An amenable equivalence relation is generated by a single transformation, Ergod. Th. \& Dynam. Sys. 1 (1981), 431-450.

[Da1] A. I. Danilenko, Comparison of cocycles of measured equivalence relations and lifting problems, Ergod. Th. \& Dynam. Sys. 18 (1998), 125-151.

[Da2] , Quasinormal subrelations of ergodic equivalence relations, Proc. Amer. Math. Soc. 126 (1998), 3361-3370.

[Da] _ Entropy theory from orbital point of view, Monatsh. Math. (to appear).

[DaG] A. I. Danilenko and V. Ya. Golodets, Extension of cocycles to normalizer elements, outer conjugacy and related problems, Trans. Amer. Math. Soc. 348 (1996), 4857-4882.

[Dy] H. A. Dye, On groups of measure preserving transformations. I, Amer. J. Math. 81 (1959), 119-159.

[FM] J. Feldman and C. C. Moore, Ergodic equivalence relations, cohomology, and von Neumann algebras. I, Trans. Amer. Math. Soc. 234 (1977), 289-324.

[Fi] A. Fieldsteel, Relative isomorphism theorem for Bernoulli flows, Isr. J. Math. 40 (1981), $197-216$.

[FiH] A. Fieldsteel and J. R. Hasfura-Buenago, Dyadic equivalence to completely positive entropy, Trans. Amer. Math. Soc. 350 (1998), 1143-1166.

[GTW] E. Glasner, J.-P. Thouvenot and B. Weiss, Entropy theory without a past, Erg. Th. \& Dynam. Sys. 20 (2000), 1355-1370.

[Ho] C. Hoffman, The behavior of Bernoulli shifts relative to their factors, Ergod. Th. \& Dynam. Sys. 19 (1999), 1255-1280.

[KaW] Y. Kazsnelson and B. Weiss, Commuting measure preserving transformation, Isr. J. Math. 12 (1972), 161-173. 
[Ki] J. C. Kieffer, A generalized Shannon-McMillan theorem for the action of an amenable group on a probability space, Ann. Prob. 3 (1975), 1031-1037.

[KW] Y. Kifer and B. Weiss, Generating partitions for random transformations, Preprint.

[Kr] W. Krieger, On entropy and generators of measure preserving transformations, Trans. Amer. Math. Soc. 149 (1970), 453-464, Erratum: 168(1972), 519.

[Li] D. A. Lind, The structure of a skew products with ergodic group automorphisms, Isr. J. Math. 28 (1977), 205-248.

[Lo] M. Loève, Probability theory. II, Fourth edition. Graduate Texts in Mathematics, Vol. 46, Springer-Verlag, New York, Heidelberg, Berlin, 1978.

[Ol] J. M. Ollagnier, Ergodic theory and statistical mechanics, Lecture Notes in Math., 1115, Springer-Verlag, Berlin, Heidelberg, New York, 1985.

[Or1] D. Ornstein, Two Bernoulli shifts with the same entropy are isomorphic, Adv. in Math. 4 (1970), 337-352.

[Or2] - Bernoulli shifts with infinite entropy are isomorphic, Adv. in Math. 5 (1970), 339-348.

[OrS] D. S. Ornstein and P. Shields, An uncountable family of K-automorphisms, Adv. in Math. 10 (1973), 63-88.

[OrW] D. S. Ornstein and B. Weiss, Entropy and isomorphism theorems for actions of amenable groups, J. d'Analyse Math. 48 (1987), 1-141.

[Rok] V. A. Rokhlin, Lectures on the entropy theory of transformations with invariant measure, Russian Math. Surveys 22, No 5 (1967), 1-52.

[Ro] A. Rosenthal, Finite uniform generators for ergodic, finite entropy, free actions of amenable groups, Probab. Th. Rel. Fields 77 (1988), 147-166.

[Pa] K. K. Park, Three Bernoulli factors that generate an ergodic flow, Dynamical systems (College Park, MD, 1986-87), 608-616, Lecture Notes in Math., 1342, Springer-Verlag, Berlin, 1988.

[RW] D. J. Rudolph and B. Weiss, Entropy and mixing for amenable group actions, Annals of Math. 151 (2000), 1119-1150.

[STh] M. Smorodinsky and J.-P. Thouvenot, Bernoulli factors that span a transformation, Isr. J. Math. 32 (1979), 39-43.

[Sw] L. Swanson, Generators of factors of Bernoulli shifts, Pacific J. Math. 71 (1977), 213220 .

[Th1] J.-P. Thouvenot, Quelques proprietes des systemes dynamiques qui se decomposent en un produit de deux systemes dont l'un est un schema de Bernoulli, Isr. J. Math. 21 (1975), 177-207.

[Th2] Une class de systemes pour lesquels la conjecture de Pinsker est vraie, Isr. J. Math. 21 (1975), 208-214.

[WaZ] T. Ward and Q. Zhang, The Abramov-Rokhlin entropy addition formula for amenable group actions, Mh. Math. 114 (1992), 317-329.

Department of Mathematics, College of Natural Science, Ajou University, SuWON 442-749, KOREA

E-mail address: danilenk@madang.ajou.ac.kr, and kkpark@madang.ajou.ac.kr 